\title{
MiR-21-3p Promotes Hepatocellular Carcinoma Progression Through Regulating Smad7/Yap1
}

\section{Yinghui Hong}

Wuhan University Zhongnan Hospital https://orcid.org/0000-0002-3828-2440

\section{Dan Wang}

Wuhan University Zhongnan Hospital

\section{Mingliang Ye}

Wuhan University

Chun Wang

Wuhan University

Jie Luo

Wuhan University

Jialiang Liu

Wuhan University

Jing Liu

Wuhan University Zhongnan Hospital

\section{Qiu Zhao}

Wuhan University Zhongnan Hospital

Ying Chang ( $\nabla$ changying@whu.edu.cn )

Hubei Clinical Center and Key Laboratory of Intestinal and Colorectal Diseases, Wuhan, China; https://orcid.org/0000-0001-8972-4109

\section{Primary research}

Keywords: MiR-21-3p, hepatocellular carcinoma, Smad7, Yap1, prognosis

Posted Date: November 17th, 2020

DOI: https://doi.org/10.21203/rs.3.rs-105350/v1

License: (a) (i) This work is licensed under a Creative Commons Attribution 4.0 International License. Read Full License 


\section{Abstract}

\section{Background}

Hepatocellular carcinoma (HCC) remains the global burden due to its high prevalence and mortality. Emerging evidence confirms that microRNAs (miRNAs) play a vital role in cancer initiation and progression. MiRNAs are widely involved in the regulation of signaling pathways by targeting downstream genes. MiR-21-3p as a liver-enriched miRNA has not been fully investigated. Abnormal activation of TGF- $\beta$ transduction pathway promoted by deletion of Smad7 matters since HCC occurrence. While the relation between miR-21-3p and Smad7 has not yet been confirmed. We aimed to explore the influence of miR-21-3p on HCC initiation and progression by targeting Smad7 and further facilitating the expression of Yap1.

\section{Methods}

MicroRNA (miRNA) microarray analysis was performed for miRNA screening. Dual-luciferase assay was adopted for target verifying. The expressions of miRNA and related genes were quantified by qRT-PCR, western blotting, and immunohistochemical staining. Flow cytometry and transwell assay were used to discover cell apoptosis, invasion and metastasis abilities. Rat models were established to explore the axis's role in hepatocarcinogenesis. Bioinformatics analysis was performed for analyzing clinical significance.

\section{Results}

MiR-21-3p was significantly increased in HCC, indicating a poor overall survival (OS) rate. High miR-21-3p was associated with advanced stages $(P=0.029)$, especially T stages $(P=0.026)$. Low Smad7/high Yap1 was verified in HCCs and rat models. Smad7 was proved to be the direct target of miR-21-3p. MiR-21-3p's effect on tumor malignant phenotypes and promotion of Yap 1 could be partly reversed through transfecting Smad7. Overexpressed Yap1 promoted the downstream effector connective tissue growth factor (CTGF). Co-survival analysis indicated that lower miR-21-3p/higher $\operatorname{Smad7}(P=0.0494)$ and lower miR-21-3p/lower Yap1 group ( $P=0.0379)$ patients had better OS rates. GSVA analysis of miR-21-3p and Smad7 related gene sets displayed strong relation with TGF- $\beta$ signaling pathway in HCC.

\section{Conclusions}

MiR-21-3p promotes HCC migration and invasion via directly inhibiting Smad7 and further improving the expression of Yap1.

\section{Background}

With a rising global incidence and the leading cause of cancer-related death status, hepatocellular carcinoma (HCC) is still lacking effective treatment (1). More than half of HCC patients are already in advanced stages of liver cancer when first diagnosed (2). For those patients with unresectable HCC, 
sorafenib and lenvatinib as multi-kinases inhibitors are considered as systemic efficiency drugs (3), while their performance on prolonging patient survival rate is far from satisfied (4). It is time to explore specific mechanisms of occurrence and progression of HCC to find effective clinical treatments.

MicroRNAs (miRNAs), the small endogenous non-coding ribonucleic acid molecular family, are mainly involved in RNA silencing and regulation of gene expressions through complementary combination with the $3^{\prime}-$ UTR region of genes (5). MiRNAs are considered to be biomarkers of diagnosis and prognosis since discovered (6). MiR-21-3p was demonstrated to inhibit cell apoptosis rate in liver cancer stem cells via directly regulating PTEN (7). Our miRNA microarray analysis results displayed that miR-21-3p is significantly upregulated in HCC. Previous studies confirmed that miR-21-3p could influence hepatoma cell growth through directly down-regulating adenosyltransferases 2A and 2B (MAT2A/MAT2B) (8). However, more important targets wait to be identified to elucidate the indispensable role of miR-21-3p in HCC.

Smad7 (I-Smad) is the negative regulator of the TGF- $\beta$ signaling transduction pathway through binding TGF- $\beta$ Receptor $\otimes(T \beta R \otimes)$ and interfering with the recruitment of Smad2/Smad3 (R-smad), blocking functional Smad complex from interacting with DNA in the nucleus $(9,10)$. As a "double-edged sword" involving in maintaining cell normal proliferation and differentiation, the TGF- $\beta$ signaling pathway plays an irreplaceable role in tumor formation and progression (11). Dysfunction of Smad7 and excessive activation of the TGF- $\beta$ signaling pathway were commonly seen in various kinds of cancers $(12,13)$. T Feng's team has verified that the deletion of Smad7 could accelerate the progress of HCC in mice models through activating the STAT3 signaling pathway (14). Loss-of function of Smad7 promoted HCC cell proliferation, accelerated G1-S transition and reduced cell apoptosis in vivo (15). The role of Smad7s abnormal deletion in HCC needs more depth researches.

Yap1 (Yes-associated protein 1) is the "star nuclear effector" of the Hippo pathway, which consists of a series of kinases (16). Yap 1 is in hyperactive status and is considered as an oncogene in several kinds of solid tumors, including liver cancer (17). The first discovery of the crosstalk between Hippo and the TGF- $\beta$ signaling pathway was that cytoplasmic Yap1 protein helped inhibit part of the signal transduction of TGF- $\beta$ in COS-7 cell ( a CV-1 African green monkey fibroblast cell line by transformation with a mutant strain of Simian Virus 40 (SV40)) via enhancing Smad7 binding to T $\beta R \rrbracket$, and Yap1 was demonstrated to be a novel Smad7-interacting protein (18). Nevertheless, the close relationship between Smad7 and Yap1 in $\mathrm{HCC}$ remains unclear.

The present study described the overexpression of miR-21-3p in human HCC. We first proposed that MiR21-3p could facilitate the expression of Yap1 and promote malignant phenotypes progression via directly targeting Smad7 in HCC.

\section{Materials And Methods}

\section{Clinical tissues and cell culture}


Ten paired HCC tissues and corresponding background livers (BLs) were obtained from HCC patients undergoing partial hepatectomy between January 2019 and December 2020. After surgical resection, the specimens were immediately snap-frozen in liquid nitrogen. RNA-later (\#AM7201, Thermofisher, USA) was added to the cryogenic vials to prevent RNA degradation for subsequent extraction. The collection and usage of these samples were followed ethical and institutional guidelines and implement it after organizing informed consent from donors. The study was approved by the local Research Ethics Committee at Zhongnan Hospital of Wuhan University (Approval No.2018078). Cell lines (L02, Huh7, HepG2, HCCL-M3) involved in this experiment were purchased from Stem Cell Bank, Chinese Academy of Sciences. Mixture of Dulbecco's Modified Eagle's Medium (Gibco, USA) and 10\% fetal bovine serum (Gibco, Thermo Fisher, USA) and 1\% penicillin-streptomycin (Gibco, USA) was used to culture cells. All cells were grown in humidified $5 \% \mathrm{CO}_{2}$ at $37^{\circ} \mathrm{C}$.

\section{MiRNA microarray analysis}

The microarray data adopted for analysis have been described in detail before (19). Data have been uploaded in NCBI's Gene Expression Omnibus (GEO) public database http://www.ncbi.nlm.nih.gov/geo/, accession number, GSE20077).

\section{RNA extraction, Reverse transcription and quantitative real- time PCR}

Total RNA extraction from tissues and cells were using Triol Reagent (Invitrogen, USA) following the instructions. The quality and concentration of RNA were detected by Nanodrop 2000 (ThermoFisher, USA). cDNA was obtained through a reverse transcription reaction with kits (Toyobo, Japan). The expression of miRNAs and genes was examined using UltraSYBR Mixture (CWbio, Beijing, China) on the CFX96Touch Real-Time PCR system (Biorad, USA). The mRNA levels of miR-21-3p and genes were normalized to U6 (Ribobio, Guangzhou, China) and GAPDH, respectively. The relative expression ratio was calculated with the $2^{-\triangle \triangle C q}$ method. Primers used listed here:

\section{Smad7}

5'-TTCAGGACCAAACGATCTGCG-3'(sense);

5'-GATGGTGGTGACCTTTGGCAC-3'(antisense);

\section{Yap1}

5'-GAACTCGGCTTCAGGTCCTC-3'(sense);

5'- GGTTCATGGCAAAACGAGGG-3'(antisense);

\section{LATS2}

5'-TGGAATGCCAACAATGTAGCG-3' (sense); 
5'-ATTATCACTCTCTCCAGGGGCG-3' (antisense);

\section{CTGF}

5'- AGAGGGCTGTCGGCG-3' (sense)

5'-CACAGGAGCTGGTGTTCCAT-3' (antisense);

\section{GAPDH}

5'-AGAAGGCTGGGGCTCATTTG-3'(sense);

5'- GCAGGAGGCATTGCTGATGAT-3'(antisense).

\section{MiRNA related reagents, plasmids and cell transfection}

The precursor and inhibitor of miR-21-3p, mimic-NC and inhibitor-NC were obtained from Ribobio (Guangzhou, China). Inhibitors of miR-21-3p were the complementary strand of miR-21-3p and was dedicated to compete the binding sites between miR-21-3p and target genes, but would not decrease the mRNA expression level of miRNAs. The final concentration of these reagents was $50 \mathrm{nM}$. Smad7 expression vector (P-Smad7) was constructed using GV141 vector by Genechem (Shanghai, China) and plasmids-NC (P-NC) served as an experimental control. All transfection experiments were performed using Lipofectamine 2000 (Invitrogen, Carlsbad, CA) following the guidance.

\section{Western blot}

Proteins collection using lysis buffer (Byotime, China) containing protease inhibitor (PMSF, 1:100). Centrifuged at $15000 \mathrm{rpm}, 20 \mathrm{~min}$ at $4^{\circ} \mathrm{C}$ and then transferring supernatant to new tubes. Concentrations of protein were detected using a BCA protein kit (Byotime, China). Mixing lysis buffer with protein loading buffer, denaturing protein at $100^{\circ} \mathrm{C}$ for $5 \mathrm{~min}$. An equal amount of proteins were separated by SDSpolyacrylamide gels and then transferred to polyvinylidene fluoride (PVDF) membranes (Millipore, USA). Blocking for $2 \mathrm{~h}$ with $5 \%$ skimmed milk. The membrane was incubated with primary antibodies overnight at $4^{\circ} \mathrm{C}$. Then reacted with second antibodies for $1.5 \mathrm{~h}$ at room temperature. The protein bands were captured by enhanced chemiluminescence (ECL) kit (Servicebio, Wuhan, China) using GENESys ( Synoptics Ltd, China). Anti-Smad7 (\#25840-1-AP), anti-Yap1 (\#14074), anti-E-cad (\#3195), anti-N-cad (\#131161), anti-Vimentin (\#5741), anti-LATS2 (\#5888) and anti-CTGF (\#86641) were purchased from CST (USA). Anti-Bcl2 (\#ab182858), anti-Bax (\#ab32503) and anti-GAPDH (\#ab8245) were purchased from Abcam. Goat anti-mouse IgG-horseradish peroxidase (\#A23220) and goat anti-mouse lgGhorseradish peroxidase (\#A25012) were come from Abbkine.

\section{Dual-luciferase reporter assay}

PmiR-GLO-Smad7 vector was constructed, which consisted of predicted binding sites that were mutated and ligated between the Pmel and Xbal restriction enzymes sites of pmirGLO Dual-Luciferase miRNA Target Reporter Vector (\#E1300, Promega, USA). Smad73'UTR region contains two putative binding sites 
for miR-21-3p with the seed regions at 1428-1434 and 1445-1451. The strand of mutant 3 (Mut 3) mutated two binding sites simultaneously. The wild-type (WT) and mutant Smad73'-UTR luciferase reporter plasmids $(0.25 \mu \mathrm{g} /$ well) were co-transfected into cell huh-7 with miR-21-3p mimics or miR-NC ( $50 \mathrm{nM}, 0.15 \mu \mathrm{l} / \mathrm{well}$ ) respectively. Dual-Glo Luciferase Assay System (\#E2920, Promega, USA) was employed to check firefly luciferase and renilla luciferase activity after $24 \mathrm{~h}$ transfection, respectively. Firefly luciferase activity was normalized to renilla luciferase activity. Data were collected using Enspire 2300 (PerkinElmer). Six repetitions per group were calculated.

\section{Cell apoptosis assay and flow cytometry}

A total of $4 \times 10^{5}$ cells were transfected with miR-21-3p mimics with/or Smad7 plasmids. After $24 \mathrm{~h}$, collecting (including dead cells in the culture medium) and processing cells according to the instructions of Annexin V-FITC/PI Double stain apoptosis detection kit (\#4101-2, Bestbio, Shanghai, China). Collecting the fluorescence intensity on the flow cytometer to check the apoptotic rate (Cytoflex Beckman, China) immediately.

\section{Cell invasion and metastasis assay}

The procedure for processing cell Huh-7 was the same as the cell apoptosis experiment. Huh-7 was harvested at $24 \mathrm{~h}$ post-transfections. Suspending cells with $5 \%$ FBS culture medium and seeding $5 \times 10^{4}$ cells each upper chamber ( $6.5 \mathrm{~mm}$. in diameter, 8.0 um pore size, Corning, USA). The lower chamber was filled with $600 \mu \mathrm{l}$ medium (15\% FBS). Cells were fixed with $4 \%$ paraformaldehyde $20 \mathrm{~min}$ at room temperature, stained with $0.1 \%$ crystal violet (Sigma-Aldrich, USA). The upper cells were wiped using cotton swabs. Selecting $100 \times$ and $200 \times$ fields and taking photos randomly under an inverted microscope (Olympus IX3). The difference between cell invasion and migration assay was that in the latter one upper chamber needed to be pretreated with $0.3 \%$ Matrigel matrix (\#356234, Corning, USA), $37^{\circ} \mathrm{C}, 4 \mathrm{~h}$. Three independent experiments were performed.

\section{Animal experiments}

The establishment of Wistar rat models were detailed descripted before, and grouping of these rats were according to the Metavir score system $(20,21)$. Here was brief introduction. Wistar rats ( male, 7-8 weeks old, 200-220 g) were purchased from Beijing Vital River Laboratory Animal Technology Co., Ltd. All animal handling and experimental procedures were approved by the Animal Care and Use Committee of Wuhan University following the Animal Experiment/Animal Biosafety Level-III Laboratory Guidelines. Rats were injected $40 \%$ carbon tetrachloride (CCl4) dissolved in maize oil $(1.5 \mathrm{ml} / \mathrm{kg})$ every twice a week. At 8 , 14, 18 weeks, liver fibrosis, cirrhosis and liver cancer were formed, respectively. Hematoxylin-eosin (HE) staining and masson staing which is more conductive to observe the degree of fiber crosslinking of rat models were displayed in Figure S1A.

\section{Immunohistochemistry (IHC)}

Immunohistochemical staining of patient tissues and animal tissues was performed by Servicebio Company, Wuhan, China, and photographed by an inverted microscope (Olympus IX3). Positively stained 
cells were counted in at least five fields from each area with $400 \times$ magnifications. Primary antibodies listed here:

Anti-Smad7: (\#25840-1-AP, Proteintech): 1:200; anti-Yap1 (\#14074, CST): 1:400.

\section{Immunofluorescence (IF)}

The distribution of microfilaments and cytoskeleton was clearly shown through fluorescently labeled phalloidin staining. Cells were cultured in soft and still matrixes $24 \mathrm{~h}$. Fixed with $4 \%$ paraformaldehyde $20 \mathrm{~min}$ at room temperature. Phalloidin dilution rate: $1: 500$. Incubation at $37^{\circ} \mathrm{C}$ for $1 \mathrm{~h}$. Nuclear staining with DAPI for $5 \mathrm{~min}$. Images were processed with an inverted microscope (Olympus IX3).

Gene set variation analysis (GSVA) and Kyoto Encyclopedia of Genes and Genomes (KEGG) pathways analysis with miR-21-3p/Smad7 expression

GSVA was a useful tool provided by R (3.5.2) to explore the potential biological process and enriched KEGG pathways of research objects according to its high or low expression. The results were presented in the form of intuitive volcano maps and specific heat maps. Gene terms with $|\log F C| \geq 0.1$ and $P<0.05$ were considered statistically significant. The KEGG gene sets (c2.cp.kegg.v6.2.symbols.gmt) downloaded from the Molecular Signatures Database-MsigDB (http://www.broad.mit.edu/gsea/msigdb/index.jsp) were used for enrichment analysis.

\section{Bioinformatics data}

MicroRNA Target Prediction Database (miRDB), PicTar and TargetScan databases were used for searching miRNA targets and predicting binding sites. Cohort data including 376 HCC patients RNA-seq and clinical characteristics were downloaded from The Cancer Genome Atlas (TCGA) database (https://portal.gdc.cancer.gov/). Data with incomplete clinical information were excluded during analysis.

\section{Statistical analysis}

Data were presented here as mean \pm standard deviation (SD) and analyzed by the Student's t-test or oneway ANOVA. Quantitative data were representative of three experiments. Wilcoxon signed-rank test and Wilcoxon rank-sum test was adopted to analyze gene expressions in paired and non-paired tissue samples. The relationships between disease stages and gene expressions was checked by Wilcoxon rank-sum test. Prognosis analysis was performed using Kaplan-Meier method and univariate Cox regression. All bioinformatics statistical analyses and plots were produced using $\mathrm{R}$ (v.3.5.2). $P<0.05$ was considered statistically significant.

\section{Results}

MiR-21-3p was upregulated in HCC and its clinical significance in HCC based on bioinformatics analysis

Based on our previous chip analysis results, miR-21-3p was significantly upregulated in HCC compared to normal liver tissues (NLTs), which had statistical significance $(P=0.028)$ (Table 1). To further verify the 
results of chip analysis, the mRNA expression of miR-21-3p was examined in human HCC tissue samples and Wistar rat models, respectively. Results showed that miR-21-3p was significantly enriched at HCC tissues compared to background livers (BLs) (Fig.1A (a)). Its upregulation was also observed in rats with late-stage fibrosis and cirrhosis (Fig.1A (b)). Compared to human relative normal cell L02, miR-21-3p was increased in liver cancer cell lines (Huh-7, Hep-G2, HCCL-M3) (Fig.1A (c)). Considering the well expression consistency in human tissues, rat liver disease models and cell lines, miR-21-3p was selected for subsequent analysis. To explore the clinical significance of the miR-21-3p in human HCCs, the Cancer Genome Atlas (TCGA) data, including 376 HCC patients, whose survival status was available, were analyzed. We found that patients with high miR-21-3p expression were significantly correlated with advanced clinical stages $(P=0.029)$, especially in T staging $(P=0.026)$, while no noticeable difference was observed in age and gender. (Figure 1B). Higher miR-21-3p expression level was correlated to shorter ten-year overall survival (OS) time (log-rank $P=0.026$ ) (Fig. 1C). GSVA analysis of miR-21-3p displayed that high expression of miR-21-3p was mainly enriched at 20 pathways in HCC (Fig.1D). In particular, the top three gene sets related were nitrogen metabolism, fatty acid metabolism and primary bile acid biosynthesis, severally (Fig.1E). KEGG pathway analysis of miR-21-3p targets revealed that the downstream targets were strongly linked to metabolic pathways, Hippo signaling road and TGF-beta signaling pathway (Table 2). The intersections of miR-21-3p potential targets from three authoritative databases (TargentScan, PicTar, miRDB) were Smad7, HBP1, FBX011, respectively (Fig.1F). Of these, Smad7 earned the highest score (Table 3), while the potential relationship between miR-21-3p and Smad7 waited to be rectified.

\section{Smad7 as the direct target of miR-21-3p was decreased in HCC}

To further verify our analysis, expressions of Smad7 in human tissue samples and rat models were checked. Compared to BLs, Smad7 was obviously decreased in HCCs (9/10) (Fig.2A (a)). Also, the lighter brownish staining was observed in HCCs compared to BLs (Fig.2B (a)). Along with the progressing of liver disease in rat models, a gradually descending trend was observed in Smad7s protein expression level (Fig.2A (b)), and immunohistochemical staining showed the same trend (Fig.2B (b)). Considering the apparent up-regulation of miR-21-3p and down-regulation of Smad7 in HCC, Dual-Luciferase assay was done in Huh-7 cells to confirm the linear regulation relation between miR-21-3p and Smad7.

Prediction websites presented there were two binding sites in the 3'-UTR region of Smad7 with high scores. In the group of co-transfecting miR-21-3p and reporter vector containing wild-type (WT) Smad7, luciferase activity was significantly inhibited, while in the group containing mutation Smad7 reporter vectors (mutation-3 (Mut3) mutating two sites simultaneously), the inhibition efficiency was no statistical significance between miR-NC and miR-21-3p group (Fig.2C). The above results indicated that the two prediction sites might both exert the effect on inhibiting Smad7.

Overexpression of Smad7 partly abrogates the progressive tumor effect of miR-21-3p on cell malignant phenotypes in HCC 
Mimics and inhibitors of miR-21-3p were transfected into Huh-7 and Hep-G2 cells to gain and lose its function separately. Plasmids of Smad7 (P-Smad7) was constructed and transfected into Huh-7 and HepG2 to overexpress Smad7, and Plasmid-NC (P-NC) served as the negative control. Smad7 expression was promoted after transfecting miR-21-3p inhibitors and decreased following transfecting miR-21-3p mimics in Huh-7 (Fig.3A (a)) and Hep-G2 cells (Fig.3A (b)). To investigate the effect of the miR-21-3p/ Smad7 on tumor cell malignant phenotypes in $\mathrm{HCC}$, biomarkers related to cell apoptosis, migration and invasion were examined. Pro-apoptotic protein Bax and epithelial signature protein E-cadherin (E-cad) were upregulated while anti-apoptotic protein $\mathrm{Bcl}-2$, mesenchymal characteristic protein $\mathrm{N}$-cadherin ( $\mathrm{N}$-cad) and Vimentin were down-regulated after transfecting miR-21-3p inhibitors or Smad7 plasmids in Huh-7 and Hep-G2 respectively (Fig.3A-B). The effect of miR-21-3p on cell malignant biomarkers was partly attenuated by co-transfecting Smad7in both two cell lines (Fig.3B). Flow cytometry results displayed that the early apoptotic rate stained green was increased by transfecting $\operatorname{Smad} 7(P<0.01)$ and deceased by upregulating miR-21-3p ( $P<0.05)$ (Fig.4A). The migration (Fig.4B (a-b)) and invasion abilities (Fig.4B (c-d)) enhanced by transfecting miR-21-3p mimics were partly reversed by co-transfecting Smad7.

\section{MiR-21-3p could enhance the expression of Yap1 partly via down-regulating Smad7}

The KEGG pathway enrichment analysis displayed that miR-21-3p was significantly related to the Hippo signaling pathway (Table 2). Besides, a previous study confirmed that Yap1 could enhance the binding of Smad7to T $\beta R \bigotimes$ (18). To further investigate the potential link among, miR-21-3p, Smad7 and Yap1 in HCC, the expression of Yap1 was detected in HCC tissues and rat models. Compared to BLs, Yap1 was enriched at human HCC tissues (8/10) (Fig.5A), and advanced stages of liver fibrosis and cirrhosis compared to F0 (F: fibrosis) (Fig.2A (b)). The darker brownish staining in immunohistochemistry images was observed in HCC tissues (Fig.5B (a)) and the stage of F3 and F4 in rats compared to F0 (Fig.5B (b)). In both Huh-7 and Hep-G2 cells, Yap1's expression was promoted through enhancing miR-21-3p and inhibited when cells were transfecting miR-21-3p inhibitors or up-regulating Smad7 (Fig.5C (a,b)). In protein level, the promotion of expression of Yap1 was partly attenuated by co-transfecting Smad7 (Fig.5C (b)). However, in the mRNA level, the influence of miR-21-3p and Smad7 on Yap1 had no statistical significance (Fig 5C (c,d)). Large tumor suppressor 2 (LATS2) as the upstream regulator of Yap1 was decreased by up-regulating Smad7(Fig.5C(b), S1(A-B)). Besides, mRNA (Fig.S2A-B) and protein (Fig.5C(b)) expression trend of connective tissue growth factor (CTGF) which was proven to be the direct nuclear target of nuclear Yap1 was consistent with Yap1 here (22) (Fig.5C (b)). Therefore, we deduced that miR-21-3p could promote the expression of Yap1 partly through downregulating Smad7.

\section{The clinical significance of Smad7/ Yap1 based on bioinformatics analysis}

Data about 376 HCC patients was downloaded from the Cancer Genome Atlas (TCGA) database. Wilcoxon rank-sum test results displayed that Smad7 was decreased $(P=4.578 \mathrm{e}-04)$ and $Y a p 1(P=3.6 \mathrm{e}-$ 05) was increased in HCC compared to adjacent normal liver tissues (Fig.6A). Combining RNA-seq data with patients' clinical traits, low Smad7/ high Yap1 was related to low grading and staging (Fig.6B). Cosurvival analysis results indicated that Lower miR-21-3p/Higher Smad7 $(P=0.049)$ and lower miR-21- 
3p/lower Yap1 $(P=0.038)$ were related to a better five-year OS rate (Fig.6C). GSVA volcano map showed that Smad7 was mainly involved in 18 pathways (Fig.6D). Of note, TGF- $\beta$ signaling pathway, Notch signaling pathway, adherens junction were most relevant in HCC (Fig.6E).

\section{Discussion}

$\mathrm{HCC}$ as the second leading cause of cancer-related death worldwide lacks efficient treatments. Abnormal expression of miRNAs played a critical role in cancer occurrence and progress including hepatocarcinogenesis. Previous reports focused on introducing the guide strand miR-21-5p's function in HCC, few researches about the passenger strand miR-21-3p. Until recently, reports on the independent function of miR-21-3p gradually increased, which brought us a new understanding of this microRNA $(23,24)$. The independent function of miR-21-3p includes it could be used as a signature in predicting the survival rate of triple-negative breast cancer (25). Besides, it's overexpression could facilitate the pulmonary metastasis by influencing the junction between tumor and stromal cells (26). In our study, we investigated miR-21-3p's role in the occurrence and progression of HCC. MiR-21-3p was increased in HCC tissue samples compared to adjacent background livers $(P<0.001)$. Further, based on the analysis of mRNA expression level of miR-21-3p in rat models, miR-21-3p may be involved in not only HCC progression but also hepatocarcinogenesis. KEGG pathway analysis of miR-21-3p's targets indicated that it was strongly related to TGF- $\beta$ signaling transduction pathway and Hippo signaling pathway. Besides, the abnormal overexpression of miR-21-3p facilitated the malignant phenotypes of liver cancer cells.

Chronic liver diseases including liver fibrosis, cirrhosis are strongly associated with HCC (27). One in three patients with cirrhosis will develop HCC in their life (28). Dysregulation of TGF- $\beta$ signaling transduction pathway was observed and testified in liver fibrosis and cirrhosis (29). Enhancing evidence demonstrated that miRNAs were involved in the process of liver fibrosis and cirrhosis through directly targeting Smad proteins (30). Our results highlighted the role of Smad7 in HCC. The absence of Smad7 was widely seen in HCC tissues compared to BLs, and had a gradually descending trend along with the progression of liver disease in rat models. Besides, deletion of Smad7 showed a good consistency in tissues and cells in HCC. We confirmed that Smad7 was the direct target of miR-21-3p in HCC. Restoring Smad7 could impair the migration and invasion abilities which were enhanced by miR-21-3p of liver cancer cell lines Huh-7 and Hep-G2, promote cell early apoptosis. During GSVA analysis, Smad7 was mainly involved in TGF- $\beta$ signaling transduction pathway in HCC. Therefore, in the occurrence and development of HCC, the deletion of Smad7 really matters.

Previous gene sets analysis of miR-21-3p's targets confirmed that it had strong relation with Hippo signaling pathway. Yap1 was obviously enriched at HCC tissues compared to BLs. In advanced stages of liver disease in rat models, it's expression was higher than normal liver tissue. Our experiments explored that overexpression of miR-21-3p could facilitate the expression of Yap1. The ascending of Yap1 via overexpression of miR-21-3p could be partly reversed by transfecting Smad7 in the protein level. Inccreased Yap1 enhanced the expression of CTGF. Upstream regulator LATS2 was the core serine/threonine kinase of the Hippo signaling transduction pathway. Once the Hippo was activated, 
phosphorylation of LATS2 could be facilitated and then further phosphorylating Yap1, resulting in Yap1 inactivation, and there is a negative feedback between Yap1 and LATS2 (31). Expression of LATS2 in overexpression of Smad7 group was downregulated while surged through transfecting miR-21-3p. This indicated that the up-regualtion of Yap1 was not caused by inactivation of LATS2 but loss function of Smad7. Further, increased Yap1 weakened the negative influence of LATS2. Vitro experiments effect in Huh-7 cells was more pronounced than Hep-G2 cells which was correlated to the basic expressions of miR-21-3p and Smad7 in cells (Fig.1A(a), S1B). We proposed that overexpression Smad7 could cut the expression of Yap1 in the protein level, not in the mRNA level. Here we concluded the complete axis in Figure 7.

Besides, recent studies suggested that not only Yap 1 can be regulated by a series of microRNAs, but nuclear Yap1/TAZ (Yap1's paralog) could influence the conversion of pri-miRNA into pre-miRNA (32). Combining our results, we highly proposed that in the axis of miR-21-3p-Smad7/ Yap1, there is a possibility that overexpression of nuclear Yap1 could in turn affect the expression of miR-21-3p. Previous studies confirmed that Yap1 as a mechano-transduction effector translocated from cytoplasm to nucleus when cells were shifted from soft to stiff matrices (33-35). Meanwhile, during the hepatocarcinogenesis, liver fibrosis and cirrhosis are typical examples of extracellular matrix (ECM) sclerosis (36), which are the "boosters" of tumor metastasis. Although there are great differences between people, the stiff of liver cancer tissue $(55 \mathrm{kpa})$ is almost about ten times that of normal liver tissue (4kpa) detected by Fibroscan (37). A reasonable and also surprising discovery was that about twofold up-regulation of miR-21-3p was found in three cell lines (Huh-7, Hep-G2, Hep-3B) when cells were cultured on stiffer substrates (40kpa) (Fig.S3A). Besides, the nuclear Yap1 in cells cultured on stiff gels was increased (Fig.S3B). Immunofluorescence results presented the deformation of cytoskeleton and changes of cell polarity in the stiffer matrixes (Fig.S3C). This indicated that there might be a positive loop between miR-21-3p and Yap1 during the progression of HCC. However, how did cells sense the mechanical signals and then transfer them into molecular signals, even changing the level miRNAs remained unclear. Our team believed that there was still an interesting story waiting to be figured out and we're working on it.

\section{Conclusions}

Our results revealed the oncogenic role of miR-21-3p in HCC. MiR-21-3p-Smad7/ Yap1 axis was identified and might be potential therapeutic targets for HCC diagnose and treatments.

\section{List Of Abbreviations}

HCC『Hepatocellular carcinoma

MiRNAs: microRNAs

OS: overall surivival

CTGF: connective tissue growth effector 
Yap1: Yes-associated protein 1

Adenosyltransferases 2A/2B: MAT2A/MAT2B

T $\beta R \rrbracket:$ TGF- $\beta$ Receptor $\rrbracket$

GSVA: Gene set variation analysis

TCGA: The Cancer Genome Atlas

SD: standard deviation

NLT: normal liver tissues

BL: background liver

WT: wild type

ECM: extracellular matrix

\section{Declarations}

\section{Ethics approval and consent to participate}

Human tissue samples used here were approved by the local Research Ethics Committee at Zhongnan Hospital of Wuhan University (Approval No.2018078). All animal handling and experimental procedures were approved by the Animal Care and Use Committee of Wuhan University following the Animal Experiment/Animal Biosafety Level-III Laboratory Guidelines.

\section{Consent for publication}

All authors have been approved this publication.

\section{Availability of data and materials}

The datasets used and/or analysed during the current study are available from the corresponding author on reasonable request.

\section{Competing interests}

The authors declare that they have no competing interests.

\section{Funding}

This work was supported by research grants from the National Natural Science Foundation of China (No. 81670554); the Science and Technology Plan of Wuhan City (2020020601012208); the Natural Science 
Fund for Distinguished Young Scholars of Hubei Province (No. 2017CFA068) and the National Key R\&D Program of China (No. 2019YFC0121505).

\section{Authors' contributions}

HYH designed and wrote this article. HYH and WD did the experiments. YML, WC and LJ revised this manuscript and gave useful suggestions. ZQ, LJ supervised this manuscript. CY funded this article and gave useful instructions. All authors read and approved the final manuscript.

\section{Acknowledgments}

None.

\section{References}

1. Craig AJ, von Felden J, Garcia-Lezana T, Sarcognato S, Villanueva A. Tumour evolution in hepatocellular carcinoma. Nat Rev Gastroenterol Hepatol 2020;17:139-52. doi:10.1038/s41575-0190229-4

2. Yang JD, Hainaut P, Gores GJ, Amadou A, Plymoth A, Roberts LR. A global view of hepatocellular carcinoma: trends, risk, prevention and management. Nat Rev Gastro Hepat 2019;16:589-604. doi:10.1038/s41575-019-0186-y

3. Couri T, Pillai A. Goals and targets for personalized therapy for HCC. Hepatol Int 2019;13:125-37. doi:10.1007/s12072-018-9919-1

4. Kudo M, Finn RS, Qin S, Han KH, Ikeda K, Piscaglia F, et al. Lenvatinib versus sorafenib in first-line treatment of patients with unresectable hepatocellular carcinoma: a randomized phase 3 noninferiority trial. Lancet 2018;391:1163-73. doi:10.1016/S0140-6736(18)30207-1

5. Tutar L, Tutar E, Tutar Y. MicroRNAs and cancer; an overview. Curr Pharm Biotechnol 2014;15:430-7. doi:10.2174/1389201015666140519095304

6. Lin SB, Gregory RI. MicroRNA biogenesis pathways in cancer. Nature Reviews Cancer 2015;15:32133. doi:10.1038/nrc3932

7. Zhu Y, Tang H, Zhang L, Gong L, Wu G, Ni J, et al. Suppression of miR-21-3p enhances TRAILmediated apoptosis in liver cancer stem cells by suppressing the PI3K/Akt/Bad cascade via regulating PTEN. Cancer Manag Res 2019;11:955-68. doi:10.2147/CMAR.S183328

8. Slapeta J, Linares MC. Combined amplicon pyrosequencing assays reveal presence of the apicomplexan "type-N" (cf. Gemmocystis cylindrus) and Chromera velia on the Great Barrier Reef, Australia. PLoS One 2013;8:e76095. doi:10.1371/journal.pone.0076095

9. Hata A, Chen YG. TGF-beta Signaling from Receptors to Smads. Cold Spring Harb Perspect Biol 2016;8. doi:10.1101/cshperspect.a022061

10. Yan X, Liao H, Cheng M, Shi X, Lin X, Feng XH, et al. Smad7 Protein Interacts with Receptor-regulated Smads (R-Smads) to Inhibit Transforming Growth Factor-beta (TGF-beta)/Smad Signaling. J Biol 
Chem 2016;291:382-92. doi:10.1074/jbc.M115.694281

11. Batlle E, Massague J. Transforming Growth Factor-beta Signaling in Immunity and Cancer. Immunity 2019;50:924-40. doi:10.1016/j.immuni.2019.03.024

12. Zhou Q, Zhou Q, Liu Q, He Z, Yan Y, Lin J, et al. PRL-3 facilitates Hepatocellular Carcinoma progression by co-amplifying with and activating FAK. Theranostics 2020;10:10345-59. doi:10.7150/thno.42069

13. Vivekanandhan S, Mukhopadhyay D. Genetic status of KRAS influences Transforming Growth Factor-beta (TGF-beta) signaling: An insight into Neuropilin-1 (NRP1) mediated tumorigenesis. Semin Cancer Biol 2019;54:72-9. doi:10.1016/j.semcancer.2018.01.014

14. Feng T, Dzieran J, Yuan X, Dropmann A, Maass T, Teufel A, et al. Hepatocyte-specific Smad7 deletion accelerates DEN-induced HCC via activation of STAT3 signaling in mice. Oncogenesis 2017;6:e294. doi:10.1038/oncsis.2016.85

15. Wang J, Zhao J, Chu ES, Mok MT, Go MY, Man K, et al. Inhibitory role of Smad7 in hepatocarcinogenesis in mice and in vitro. J Pathol 2013;230:441-52. doi:10.1002/path.4206

16. Raj N, Bam R. Reciprocal Crosstalk Between YAP1/Hippo Pathway and the p53 Family Proteins: Mechanisms and Outcomes in Cancer. Front Cell Dev Biol 2019;7:159. doi:10.3389/fcell.2019.00159

17. Tschaharganeh DF, Chen X, Latzko P, Malz M, Gaida MM, Felix K, et al. Yes-associated protein upregulates Jagged-1 and activates the Notch pathway in human hepatocellular carcinoma. Gastroenterology 2013;144:1530-42 e12. doi:10.1053/j.gastro.2013.02.009

18. Ferrigno O, Lallemand F, Verrecchia F, L'Hoste S, Camonis J, Atfi A, et al. Yes-associated protein (YAP65) interacts with Smad7 and potentiates its inhibitory activity against TGF-beta/Smad signaling. Oncogene 2002;21:4879-84. doi:10.1038/sj.onc.1205623

19. He XX, Chang Y, Meng FY, Wang MY, Xie QH, Tang F, et al. MicroRNA-375 targets AEG-1 in hepatocellular carcinoma and suppresses liver cancer cell growth in vitro and in vivo. Oncogene 2012;31:3357-69. doi:10.1038/onc.2011.500

20. Knodell RG, Ishak KG, Black WC, Chen TS, Craig R, Kaplowitz N, et al. Formulation and application of a numerical scoring system for assessing histological activity in asymptomatic chronic active hepatitis. Hepatology 1981;1:431-5. doi:10.1002/hep.1840010511

21. Wang C, Luo J, Chen Z, Ye M, Hong Y, Liu J, et al. MiR-375 Impairs the Invasive Capabilities of Hepatoma Cells by Targeting HIF1alpha Under Hypoxia. Dig Dis Sci 2020. doi:10.1007/s10620-02006202-9

22. Kang W, Huang T, Zhou Y, Zhang J, Lung RWM, Tong JHM, et al. miR-375 is involved in Hippo pathway by targeting YAP1/TEAD4-CTGF axis in gastric carcinogenesis. Cell Death Dis 2018;9:92. doi:10.1038/s41419-017-0134-0

23. Calsina B, Castro-Vega LJ, Torres-Perez R, Inglada-Perez L, Curras-Freixes M, Roldan-Romero JM, et al. Integrative multi-omics analysis identifies a prognostic miRNA signature and a targetable miR-213p/TSC2/mTOR axis in metastatic pheochromocytoma/paraganglioma. Theranostics 2019;9:494658. doi:10.7150/thno.35458 
24. Moscetti I, Cannistraro S, Bizzarri AR. Probing direct interaction of oncomiR-21-3p with the tumor suppressor p53 by fluorescence, FRET and atomic force spectroscopy. Arch Biochem Biophys 2019;671:35-41. doi:10.1016/j.abb.2019.05.026

25. Wu X, Ding M, Lin J. Three-microRNA expression signature predicts survival in triple-negative breast cancer. Oncol Lett 2020;19:301-8. doi:10.3892/ol.2019.11118

26. Ju Q, Zhao L, Gao J, Zhou L, Xu Y, Sun Y, et al. Mutant p53 increases exosome-mediated transfer of miR-21-3p and miR-769-3p to promote pulmonary metastasis. Chin J Cancer Res 2019;31:533-46. doi:10.21147/j.issn.1000-9604.2019.03.15

27. Kanda T, Goto T, Hirotsu Y, Moriyama M, Omata M. Molecular Mechanisms Driving Progression of Liver Cirrhosis towards Hepatocellular Carcinoma in Chronic Hepatitis B and C Infections: A Review. Int J Mol Sci 2019;20. doi:10.3390/ijms20061358

28. El-Serag HB. Hepatocellular carcinoma. N Engl J Med 2011;365:1118-27. doi:10.1056/NEJMra1001683

29. Xu F, Liu C, Zhou D, Zhang L. TGF-beta/SMAD Pathway and Its Regulation in Hepatic Fibrosis. J Histochem Cytochem 2016;64:157-67. doi:10.1369/0022155415627681

30. Yang YZ, Zhao XJ, Xu HJ, Wang SC, Pan Y, Wang SJ, et al. Magnesium isoglycyrrhizinate ameliorates high fructose-induced liver fibrosis in rat by increasing miR-375-3p to suppress JAK2/STAT3 pathway and TGF-beta1/Smad signaling. Acta Pharmacol Sin 2019;40:879-94. doi:10.1038/s41401-018-0194-4

31. Taha Z, Janse van Rensburg HJ, Yang X. The Hippo Pathway: Immunity and Cancer. Cancers (Basel) 2018;10. doi:10.3390/cancers 10040094

32. Han Y. Analysis of the role of the Hippo pathway in cancer. J Transl Med 2019;17:116. doi:10.1186/s12967-019-1869-4

33. Burnette FS, Flick GJ, Jr. Comparison of Three Assays for Peroxidase in the Blue Crab ( Callinectes sapidus ). J Food Prot 1977;40:854-6. doi:10.4315/0362-028X-40.12.854

34. Halder G, Dupont S, Piccolo S. Transduction of mechanical and cytoskeletal cues by YAP and TAZ. Nat Rev Mol Cell Biol 2012;13:591-600. doi:10.1038/nrm3416

35. Tse JR, Engler AJ. Preparation of hydrogel substrates with tunable mechanical properties. Curr Protoc Cell Biol 2010;Chapter 10:Unit 10 6. doi:10.1002/0471143030.cb1016s47

36. Zeng Z, Guan L, An P, Sun S, O'Brien SJ, Winkler CA, et al. A population-based study to investigate host genetic factors associated with hepatitis $B$ infection and pathogenesis in the Chinese population. BMC Infect Dis 2008;8:1. doi:10.1186/1471-2334-8-1

37. Masuzaki R, Tateishi R, Yoshida H, Sato T, Ohki T, Goto T, et al. Assessing liver tumor stiffness by transient elastography. Hepatol Int 2007;1:394-7. doi:10.1007/s12072-007-9012-7

\section{Tables}


Table 1. Different expressions of miRNAs in all 3 cases of HCC tissues and normal liver tissues. (Top fifteen listed here)

\begin{tabular}{|llll|}
\hline \#Term & $\begin{array}{l}\text { LogFC } \\
\text { (HCC vs NLT) }\end{array}$ & P-value & Corrected P-Value \\
\hline hsa-miR-663 & 12.00859435 & 0.006642752 & 0.025159423 \\
\hline hsa-miR-452 & 10.42475206 & 0.044754151 & 0.096860769 \\
\hline hsa-miR-576-3p & 8.892785445 & 0.085150937 & 0.129003669 \\
\hline hsa-miR-224 & 8.397356424 & 0.002498379 & 0.018925218 \\
\hline hsa-miR-96 & 5.635504649 & 0.04323621 & 0.096860769 \\
\hline hsa-miR-216a & 3.536217114 & 0.1519771 & 0.191871088 \\
\hline hsa-miR-222 & 3.425619069 & 0.031592274 & 0.095724591 \\
\hline hsa-miR-551b & 2.443388558 & 0.360760502 & 0.364368107 \\
\hline hsa-miR-500* & 2.359130217 & 0.07579151 & 0.127790049 \\
\hline hsa-miR-500 & 2.31112225 & 0.189840985 & 0.205435066 \\
\hline hsa-miR-505* & 2.216287725 & 0.108599824 & 0.149571576 \\
\hline hsa-miR-21-3p & $\mathbf{2 . 1 8 8 7 4 1 1 9 6}$ & $\mathbf{0 . 0 0 5 9 1 4 8 8 1}$ & $\mathbf{0 . 0 2 7 7 9 0 0 4 9}$ \\
\hline hsa-miR-34b* & 2.013273752 & 0.005016725 & 0.025159423 \\
\hline hsa-miR-18a & 1.985718798 & 0.189563246 & 0.205435066 \\
\hline hsa-miR-34a & 1.440184224 & 0.002272378 & 0.018925218 \\
\hline
\end{tabular}

Footnotes: LogFC: Log fold change; HCC: hepatocellular carcinoma; NLT: normal liver tissue

Table 2. The Kyoto Encyclopedia of Genes and Genomes (KEGG) pathway enrichment analysis of miR21-3p downstream targets. (Top twelve listed here) 


\begin{tabular}{|c|c|c|c|c|c|}
\hline \#Term & ID & $\begin{array}{l}\text { Input } \\
\text { number }\end{array}$ & $\begin{array}{l}\text { Background } \\
\text { number }\end{array}$ & P-Value & $\begin{array}{l}\text { Corrected } \\
\text { P-Value }\end{array}$ \\
\hline Metabolic pathways & hsa01100 & 28 & 1243 & 0.0068 & 0.0303 \\
\hline Pathways in cancer & hsa05200 & 14 & 397 & 0.001 & 0.0110 \\
\hline MAPK signaling pathway & hsa04010 & 12 & 255 & 0.0003 & 0.0063 \\
\hline $\begin{array}{l}\text { PI3K-Akt signaling } \\
\text { pathway }\end{array}$ & hsa04151 & 12 & 342 & 0.0030 & 0.0173 \\
\hline $\begin{array}{l}\text { cGMP-PKG signaling } \\
\text { pathway }\end{array}$ & hsa04022 & 11 & 167 & $\begin{array}{l}2.89 \mathrm{E}- \\
05\end{array}$ & 0.0016 \\
\hline $\begin{array}{l}\text { Ubiquitin mediated } \\
\text { proteolysis }\end{array}$ & hsa04120 & 10 & 137 & $\begin{array}{l}2.93 \mathrm{E}- \\
05\end{array}$ & 0.0016 \\
\hline Insulin signaling pathway & hsa04910 & 10 & 139 & $\begin{array}{l}3.29 \mathrm{E}- \\
05\end{array}$ & 0.0016 \\
\hline Hippo signaling pathway & hsa04390 & 10 & 154 & $\begin{array}{l}7.42 \mathrm{E}- \\
05\end{array}$ & 0.0022 \\
\hline cAMP signaling pathway & hsa04024 & 10 & 199 & 0.0005 & 0.0085 \\
\hline HTLV-I infection & hsa05166 & 10 & 259 & 0.0034 & 0.01838 \\
\hline $\begin{array}{l}\text { TGF-beta signaling } \\
\text { pathway }\end{array}$ & hsa04350 & 6 & 84 & 0.0013 & 0.0110 \\
\hline mTOR signaling pathway & hsa04150 & 7 & 154 & 0.0060 & 0.0269 \\
\hline
\end{tabular}

Table 3. Intersections of potential targets of miR-21-3p based on analysis of three online databases

\begin{tabular}{|llll|}
\hline Target Gene & TargetScan total context score & $\begin{array}{l}\text { miRDB } \\
\text { target score }\end{array}$ & PicTar score \\
\hline Smad7 & -0.39 & 92 & 2.15 \\
\hline HBP1 & -0.15 & 531 & 2.22 \\
\hline FBXO11 & -0.23 & 58 & 2.79 \\
\hline
\end{tabular}

\section{Figures}



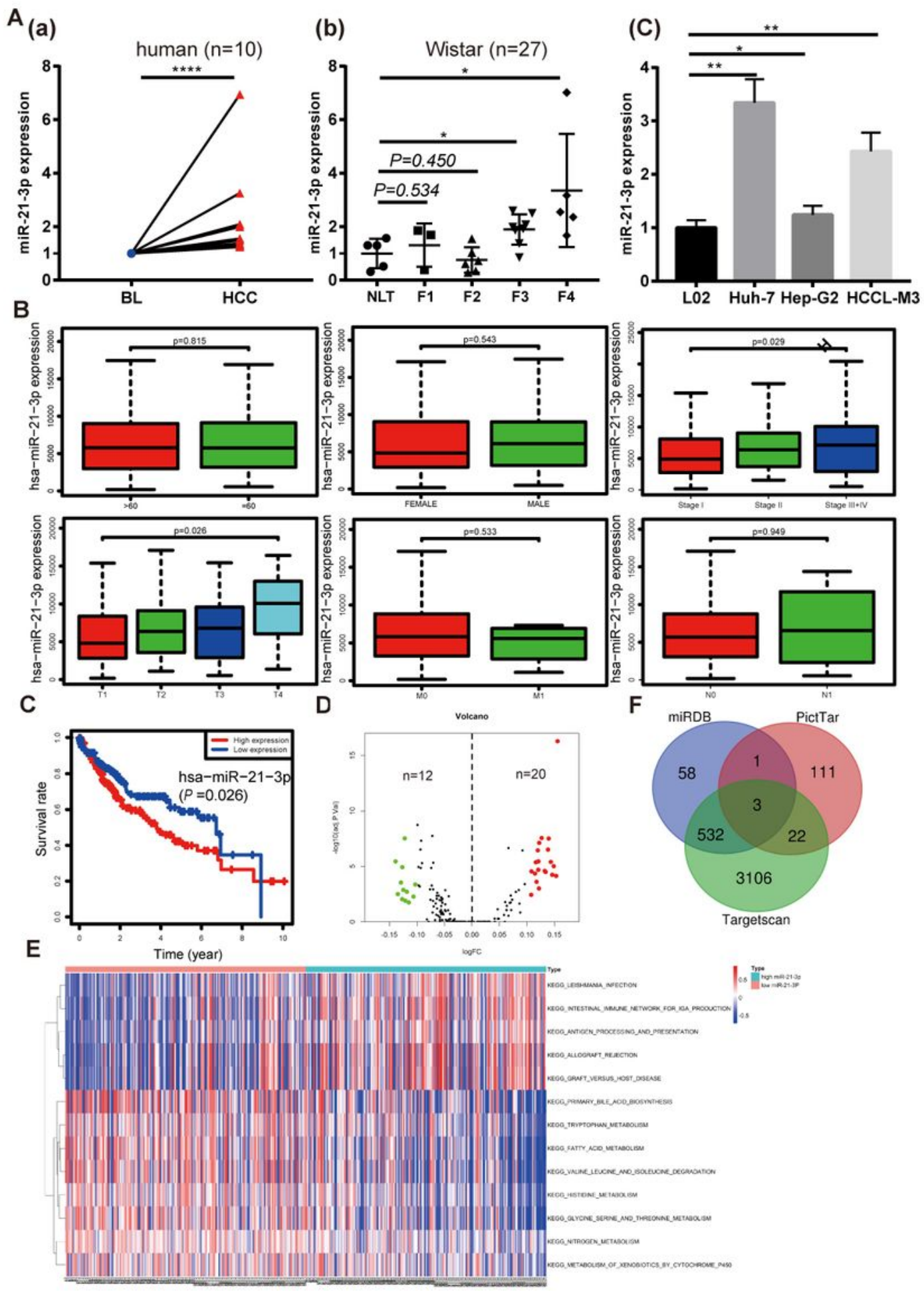

\section{Figure 1}

miR-21-3p is upregulated and its clinical significance in hepatocellular carcinoma (HCC). (A) TaqMan quantitative RT-PCR was performed to analyze microRNA-21-3p (miR-21-3p) expressions. 10 pairs of Human HCCs and their counterpart background livers (BLs) were examined (A-a); Normal, fibrosis and cirrhosis tissues from rat models were also analyzed (A-b). MiR-21-3p expression in three cell lines (L02, Huh-7 and Hep-G2) (A-c). Each dot indicates the expression level of an individual case, calculated by the 
2- $\Delta \Delta$ Ct method. (B) Association of miR-21-3p expression level with clinical characteristics (age, gender, stage, TNM staging, respectively). (C) Kaplan-Meier curves representing the relation between miR-21-3p and the percent overall survival (OS) rate in HCC patients in the TCGA data set $(n=376)$. Statistical significance between miRNA expression and OS was determined by the Log-rank test. (D) Volcano map of the gene set variation analysis (GSVA) for microarray statistics according to the high and low expression of miR-21-3p. (E) Kyoto Encyclopedia of Genes and Genomes (KEGG) pathways of miR-21-3p based on the gene set variation analysis of The Cancer Genome Atlas (TCGA) data in HCC. Significant terms were described in the heat-map (top-ten listed). (F) Venn diagram of intersections of three databases (miRDB, Targetscan, PicTar) of predicted downstream target genes of miR-21-3p. *Pख0.05, ** $\mathrm{P}$ $₫ 0.01, * \star \star \star \mathrm{P} \otimes 0.0001$. 

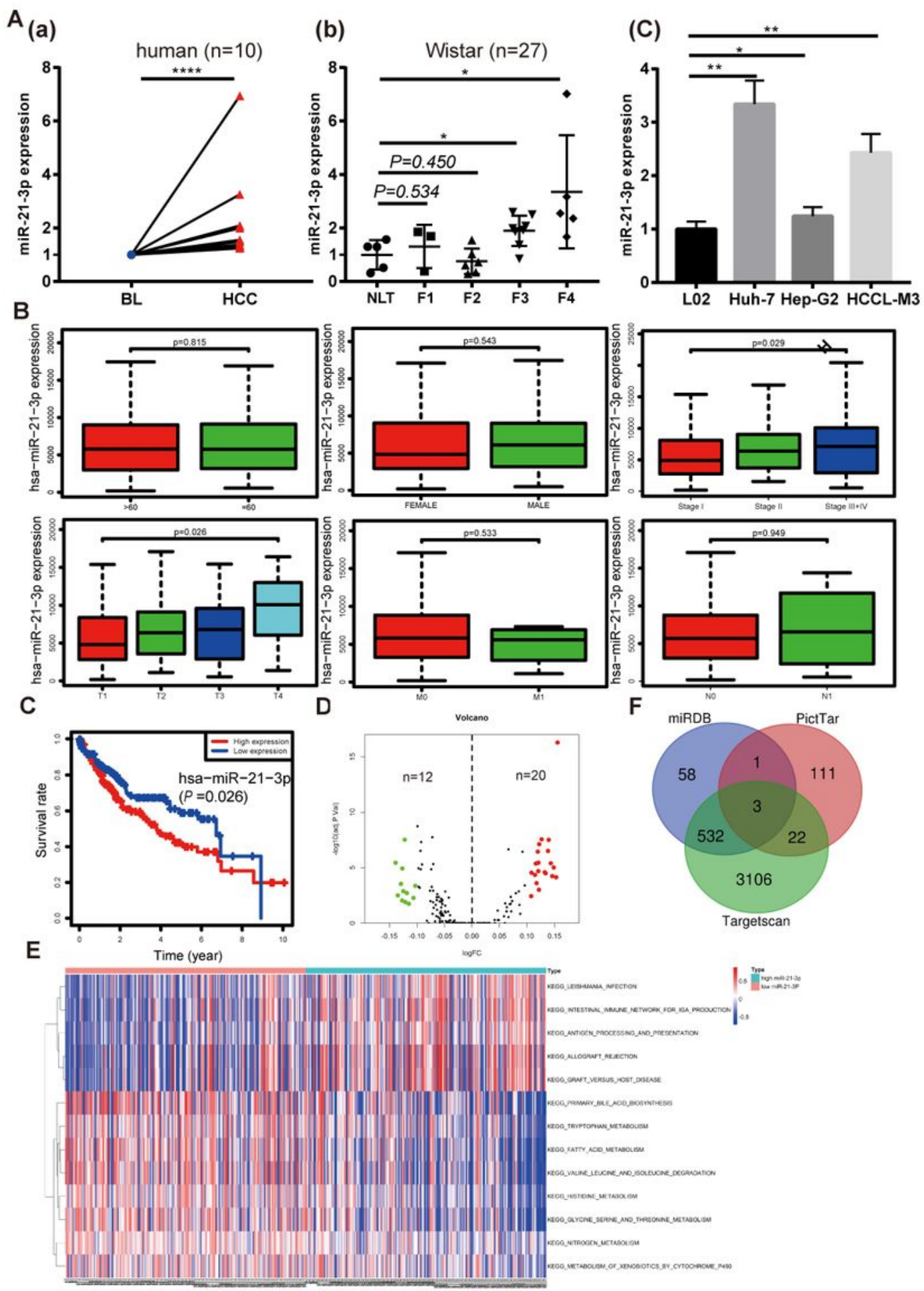

\section{Figure 1}

miR-21-3p is upregulated and its clinical significance in hepatocellular carcinoma (HCC). (A) TaqMan quantitative RT-PCR was performed to analyze microRNA-21-3p (miR-21-3p) expressions. 10 pairs of Human HCCs and their counterpart background livers (BLs) were examined (A-a); Normal, fibrosis and cirrhosis tissues from rat models were also analyzed (A-b). MiR-21-3p expression in three cell lines (L02, Huh-7 and Hep-G2) (A-c). Each dot indicates the expression level of an individual case, calculated by the 
2- $\Delta \Delta$ Ct method. (B) Association of miR-21-3p expression level with clinical characteristics (age, gender, stage, TNM staging, respectively). (C) Kaplan-Meier curves representing the relation between miR-21-3p and the percent overall survival (OS) rate in HCC patients in the TCGA data set $(n=376)$. Statistical significance between miRNA expression and OS was determined by the Log-rank test. (D) Volcano map of the gene set variation analysis (GSVA) for microarray statistics according to the high and low expression of miR-21-3p. (E) Kyoto Encyclopedia of Genes and Genomes (KEGG) pathways of miR-21-3p based on the gene set variation analysis of The Cancer Genome Atlas (TCGA) data in HCC. Significant terms were described in the heat-map (top-ten listed). (F) Venn diagram of intersections of three databases (miRDB, Targetscan, PicTar) of predicted downstream target genes of miR-21-3p. *Pख0.05, ** $\mathrm{P}$ $₫ 0.01, * \star \star \star \mathrm{P} \otimes 0.0001$. 

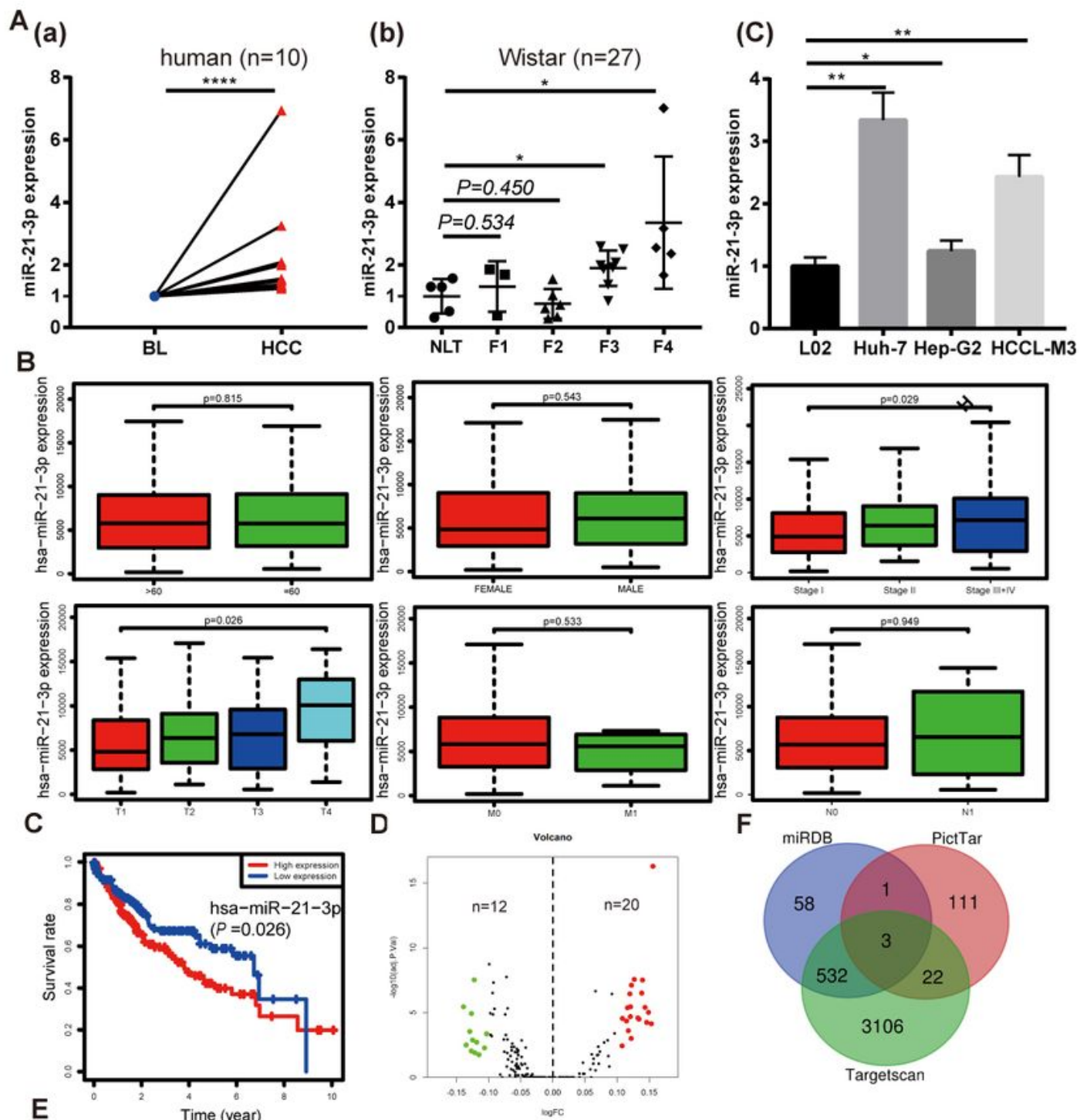

E

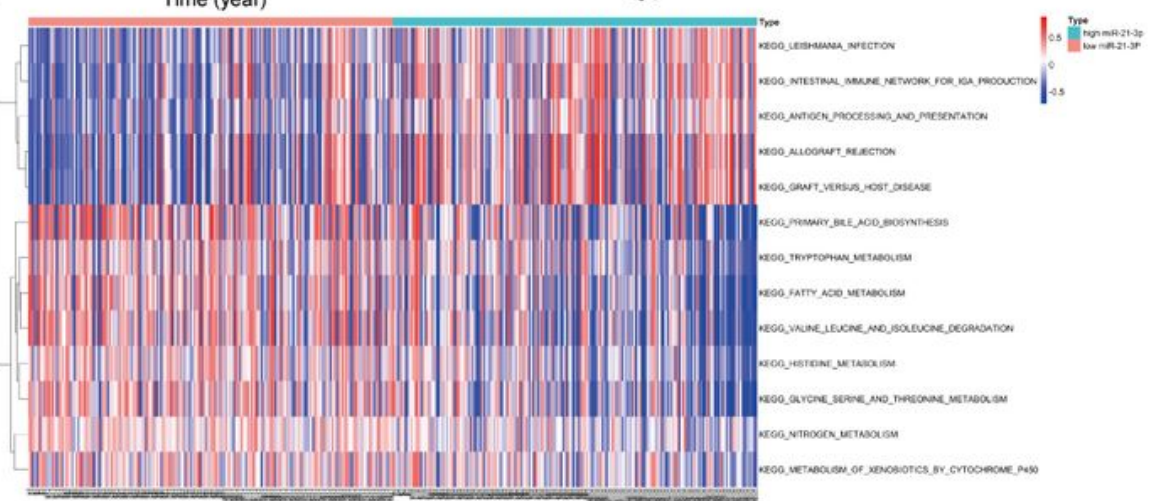

\section{Figure 2}

Smad7 was the direct target of miR-21-3p and was decreased in HCCs. (A) Western blotting and density photograph was performed to show Smad7 expressions in human HCCs (T: tumor) and their BLs (B: background) (a), different stages of rat hepatocarcinogenesis, including normal, fibrosis and cirrhosis (b). (B) Immunohistochemistry (IHC) staining images to show Smad7's expressions in HCCs and liver tissues at different stages of rat hepatocarcinogenesis (magnification 400x). Six paired human samples and 
three rats each group were calculated during statistical analysis. (C) The base pairing complement suggested the two putative miR-21-3p binding sites at 3'UTR of Yap1 predicted by TargetScan. PmiR-GLOSmad7 vector was co-transfected with mimic-21 or mimic-NC; wild-type (WT); mutation (Mut). Mut1-2 represented the two predicted binding sites, respectively. Mut-3 meant that two binding sites were mutated. Luciferase activity assay of pmiR-GLO-Smad7-3'UTR cotransfection with miR-21-3p or NC mimics. Firefly luciferase activity was measured $24 \mathrm{~h}$ after transfection and normalized to renilla luciferase activity. All data for miR-NC groups was set as 1 . Data were presented as the mean \pm SEM of the three separate triplicate experiments. ** $P \otimes 0.01$. 

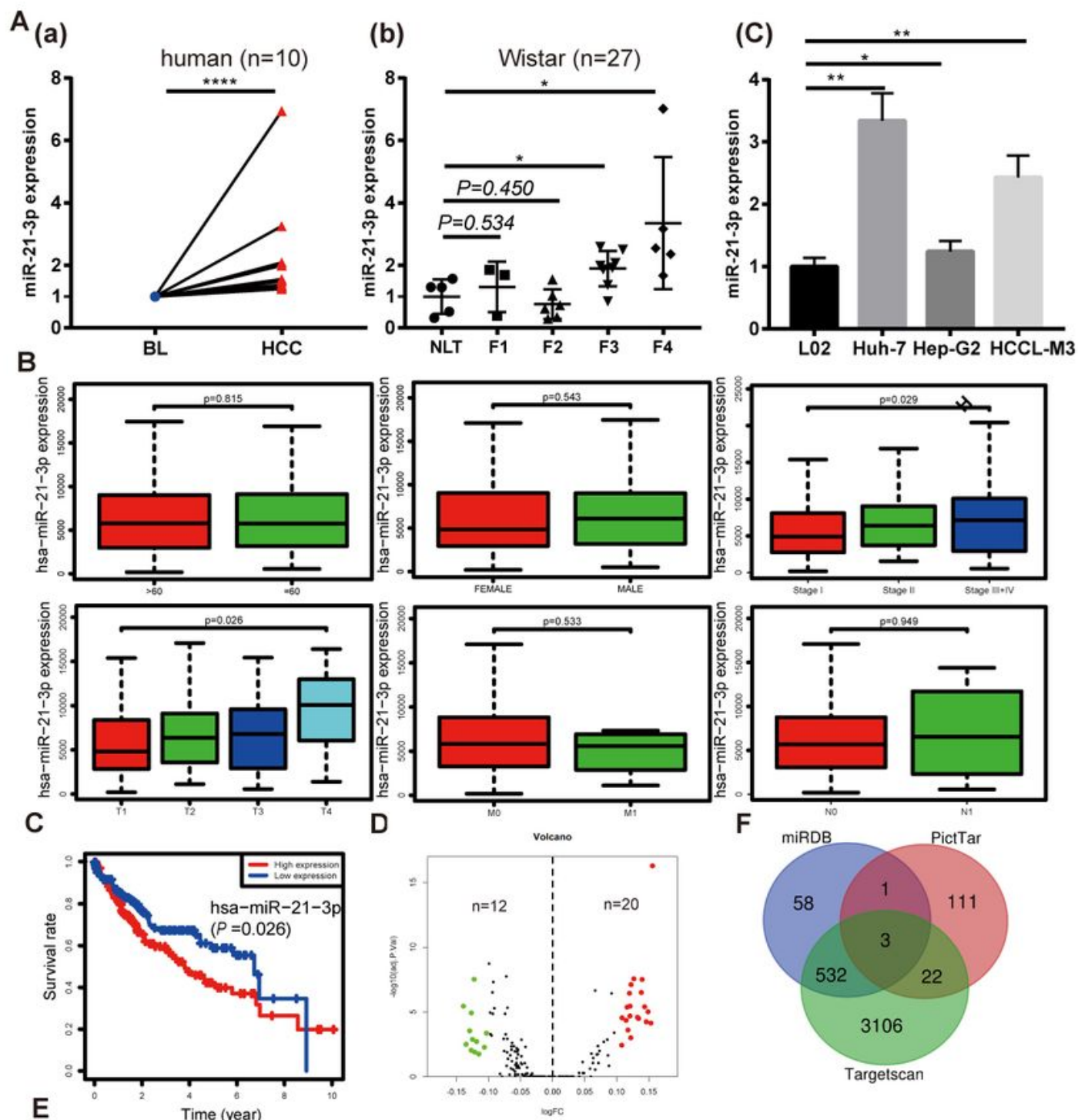

E

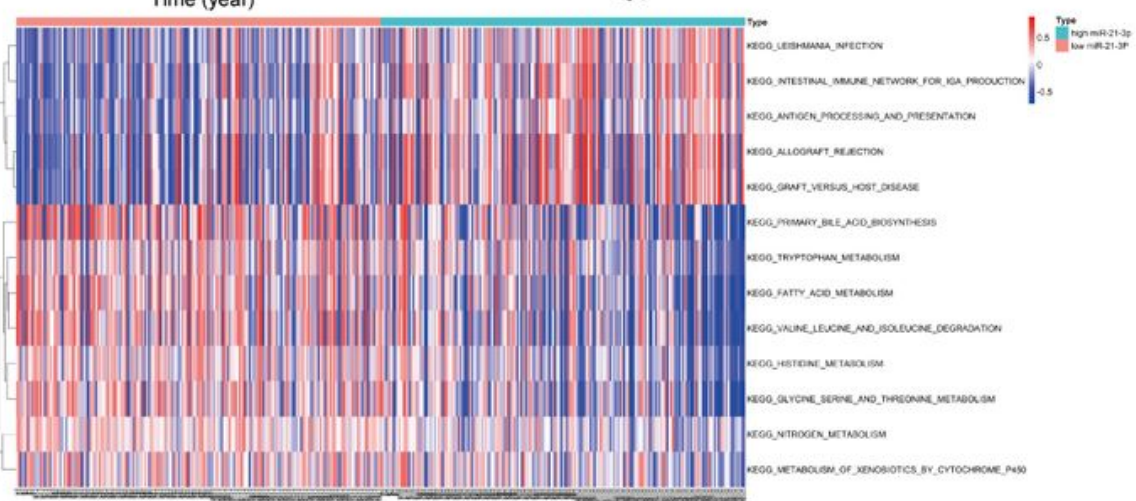

\section{Figure 2}

Smad7 was the direct target of miR-21-3p and was decreased in HCCs. (A) Western blotting and density photograph was performed to show Smad7 expressions in human HCCs (T: tumor) and their BLs (B: background) (a), different stages of rat hepatocarcinogenesis, including normal, fibrosis and cirrhosis (b). (B) Immunohistochemistry (IHC) staining images to show Smad7's expressions in HCCs and liver tissues at different stages of rat hepatocarcinogenesis (magnification 400x). Six paired human samples and 
three rats each group were calculated during statistical analysis. (C) The base pairing complement suggested the two putative miR-21-3p binding sites at 3'UTR of Yap1 predicted by TargetScan. PmiR-GLOSmad7 vector was co-transfected with mimic-21 or mimic-NC; wild-type (WT); mutation (Mut). Mut1-2 represented the two predicted binding sites, respectively. Mut-3 meant that two binding sites were mutated. Luciferase activity assay of pmiR-GLO-Smad7-3'UTR cotransfection with miR-21-3p or NC mimics. Firefly luciferase activity was measured $24 \mathrm{~h}$ after transfection and normalized to renilla luciferase activity. All data for miR-NC groups was set as 1 . Data were presented as the mean \pm SEM of the three separate triplicate experiments. ** $P \otimes 0.01$. 


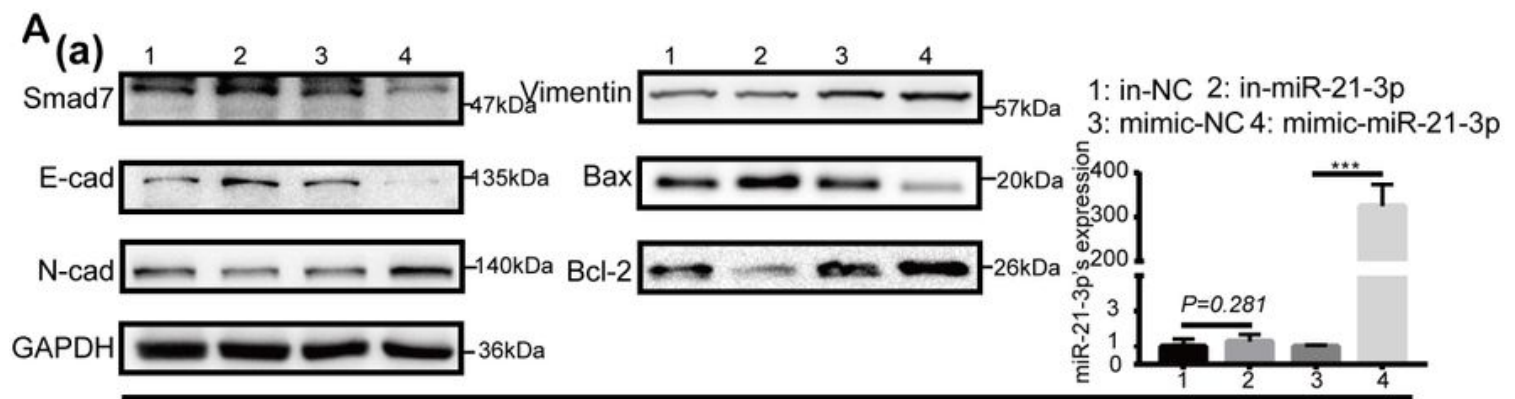

(b)
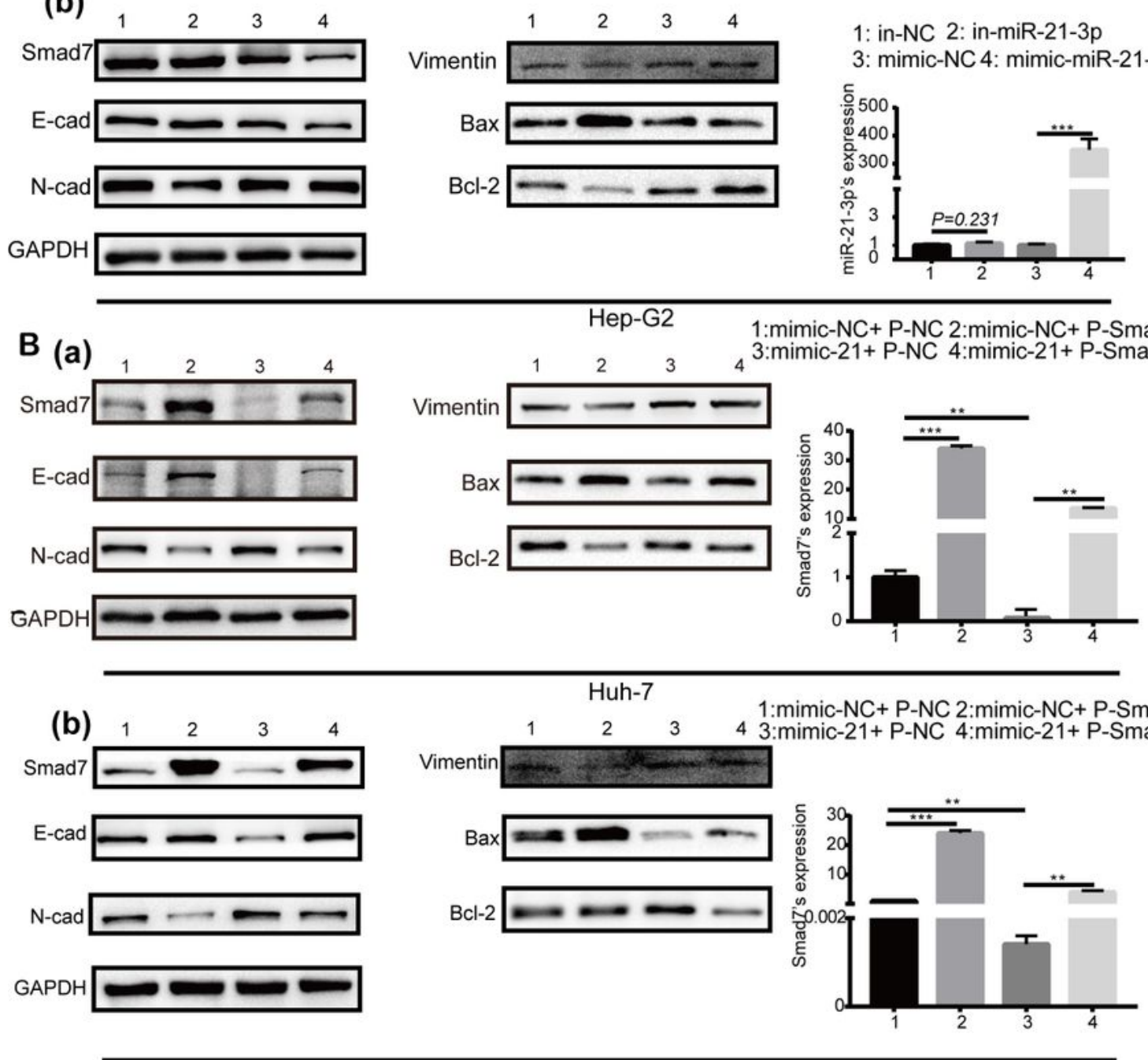

Hep-G2

\section{Figure 3}

Variations of cell phenotypes biomarkers after transfecting Smad7 with/ or miR-21-3p mimics. (A) Western blotting showed apoptotic protein Bax and Bcl-2, Epithelial-mesenchymal transition (EMT) protein E-cadherin, $\mathrm{N}$-cadherin and Vimentin expression after 48h transfection with miR-21-3p inhibitors with/or mimics in Huh-7 cells (a) and Hep-G2 cells respectively (b). Transfection effiency listed beside. (B) Plasmids-Smad7 (P-Smad7) was constructed to overexpress Smad7 and plasmids-NC (P-NC) served as 
control. Tranfection efficiency of Smad7 listed beside. Cell apoptosis (Bcl-2, Bax) and EMT biomarkers (Ecadherin, N-cadherin, Vimentin) were checked after co-transfecting P-Smad7 and/or miR-21-3p mimics in Huh-7 (a) and Hep-G2 cells (b), photo density in group1 (mimic-NC+ P-NC) was set as 1. Data were presented as the mean \pm SEM of the three separate triplicate experiments. $* * P \unrhd 0.01$, ${ }^{\star \star *} \mathrm{P} \unrhd 0.001$.
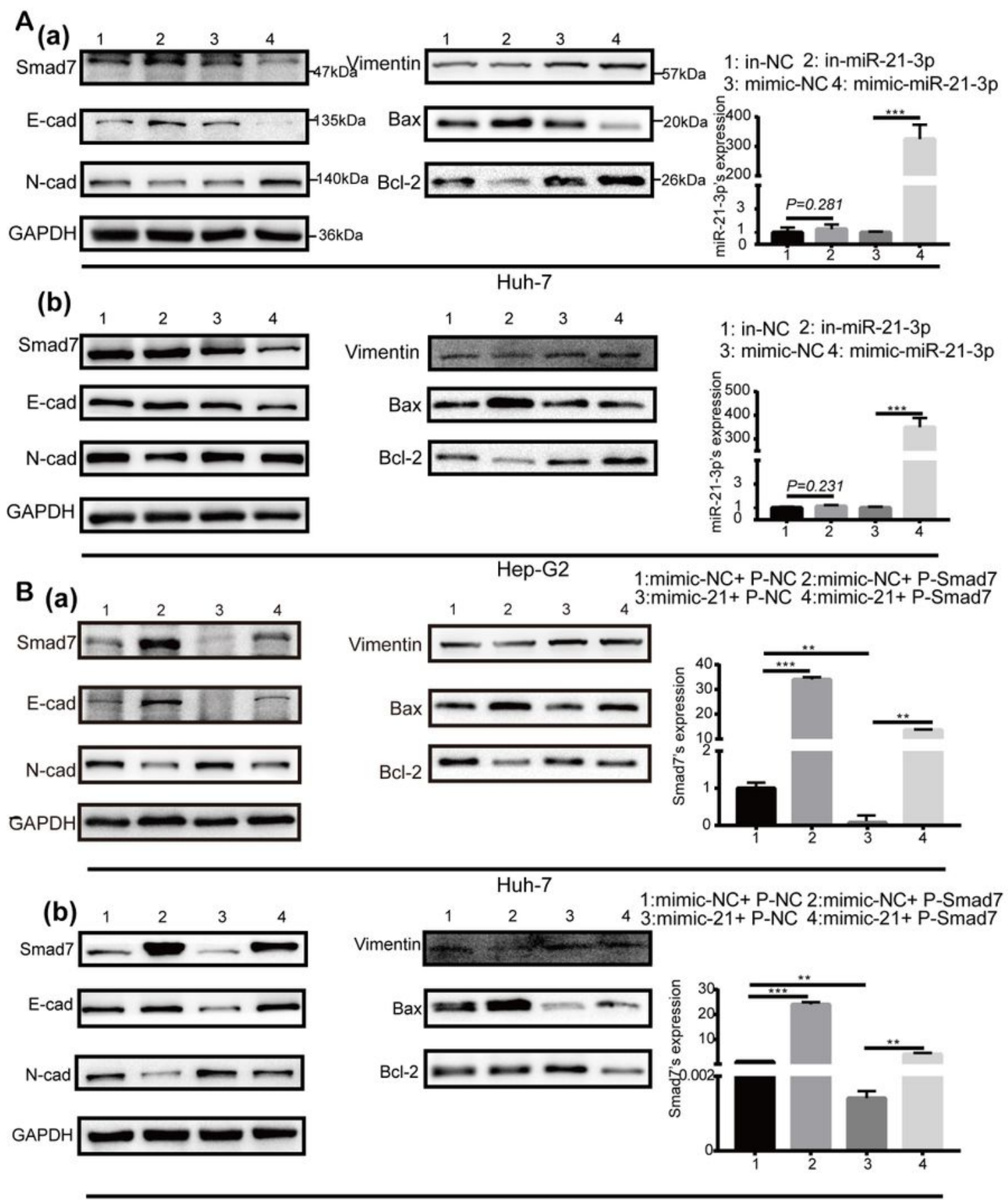

Hep-G2

Figure 3 
Variations of cell phenotypes biomarkers after transfecting Smad7 with/ or miR-21-3p mimics. (A) Western blotting showed apoptotic protein Bax and Bcl-2, Epithelial-mesenchymal transition (EMT) protein E-cadherin, N-cadherin and Vimentin expression after 48h transfection with miR-21-3p inhibitors with/or mimics in Huh-7 cells (a) and Hep-G2 cells respectively (b). Transfection effiency listed beside. (B) Plasmids-Smad7 (P-Smad7) was constructed to overexpress Smad7 and plasmids-NC (P-NC) served as control. Tranfection efficiency of Smad7 listed beside. Cell apoptosis (Bcl-2, Bax) and EMT biomarkers (Ecadherin, N-cadherin, Vimentin) were checked after co-transfecting P-Smad7 and/or miR-21-3p mimics in Huh-7 (a) and Hep-G2 cells (b), photo density in group1 (mimic-NC+ P-NC) was set as 1. Data were presented as the mean \pm SEM of the three separate triplicate experiments. ${ }^{\star \star} \mathrm{P} \otimes 0.01,{ }^{\star \star \star} \mathrm{P} \otimes 0.001$. 
A

1:mimic-NC+P-NC 2:mimic-NC+P-Smad7 3:mimic-21+P-NC 4:mimic-21+P-Smad7
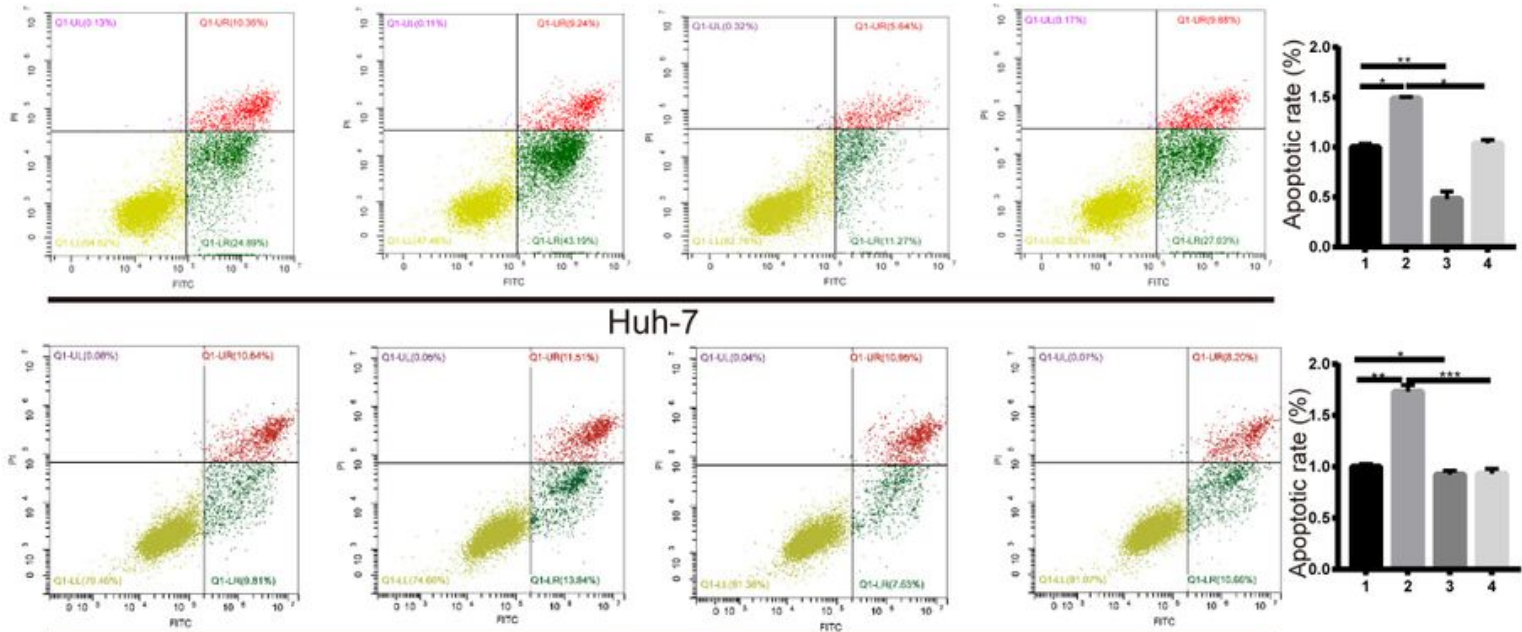

Huh-7

B
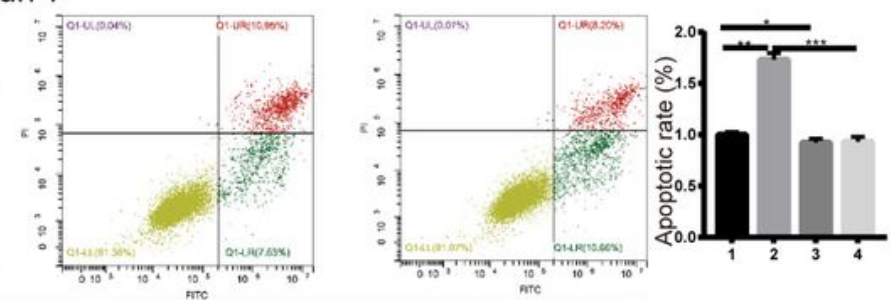

(a)
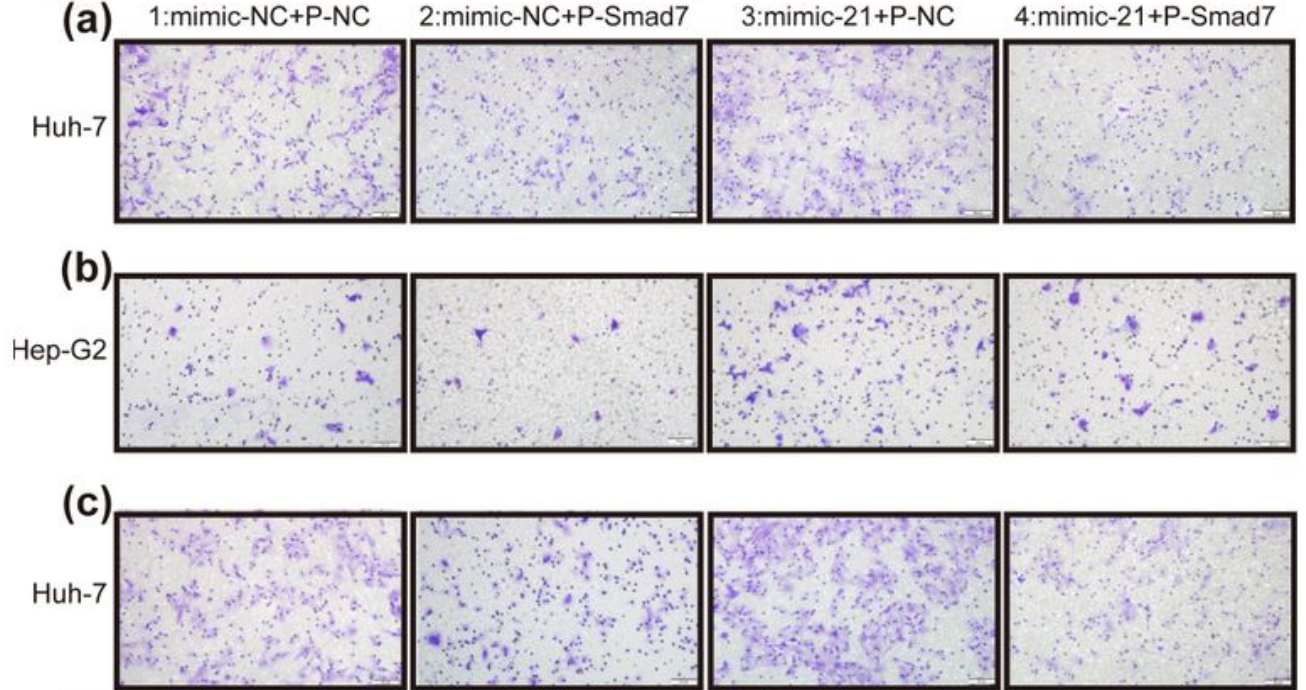

(d)
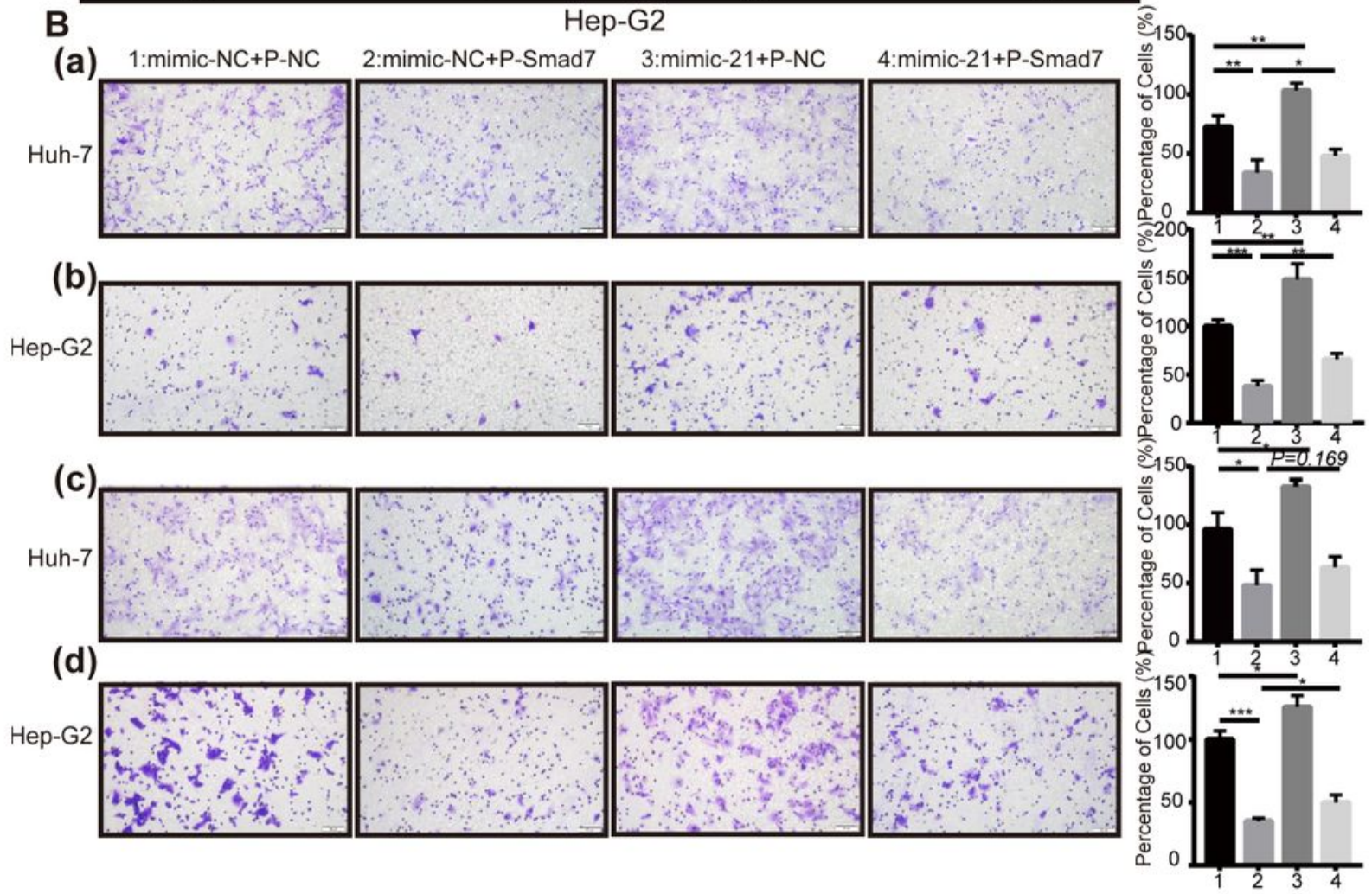

\section{Figure 4}

The effect of miR-21-3p on liver cancer cell malignant phenotypes could be partly reversed via overexpression of Smad7. (A) Flow cytometry was performed to analyze cell apoptosis index of Huh-7 and Hep-G2 cells after transfection. Cells were co-stained with Annexin FITC/PI, Annexin FITC+/PI- cells considered as early apoptotic cells were stained green. Normal, late apoptosis and necrosis cells were shown in yellow, red and pink, respectively. Data were shown as mean \pm SD from three independent 
experiments. (B) Transwell assay detected the migration (a, b) and invasion (c, d) ability of Huh-7 and Hep-G2 cells after transfection separately. ${ }^{*} P<0.05$, ** $P<0.01$; $* \star \star P<0.001$.

A

1:mimic-NC+P-NC 2:mimic-NC+P-Smad7 3:mimic-21+P-NC 4:mimic-21+P-Smad7
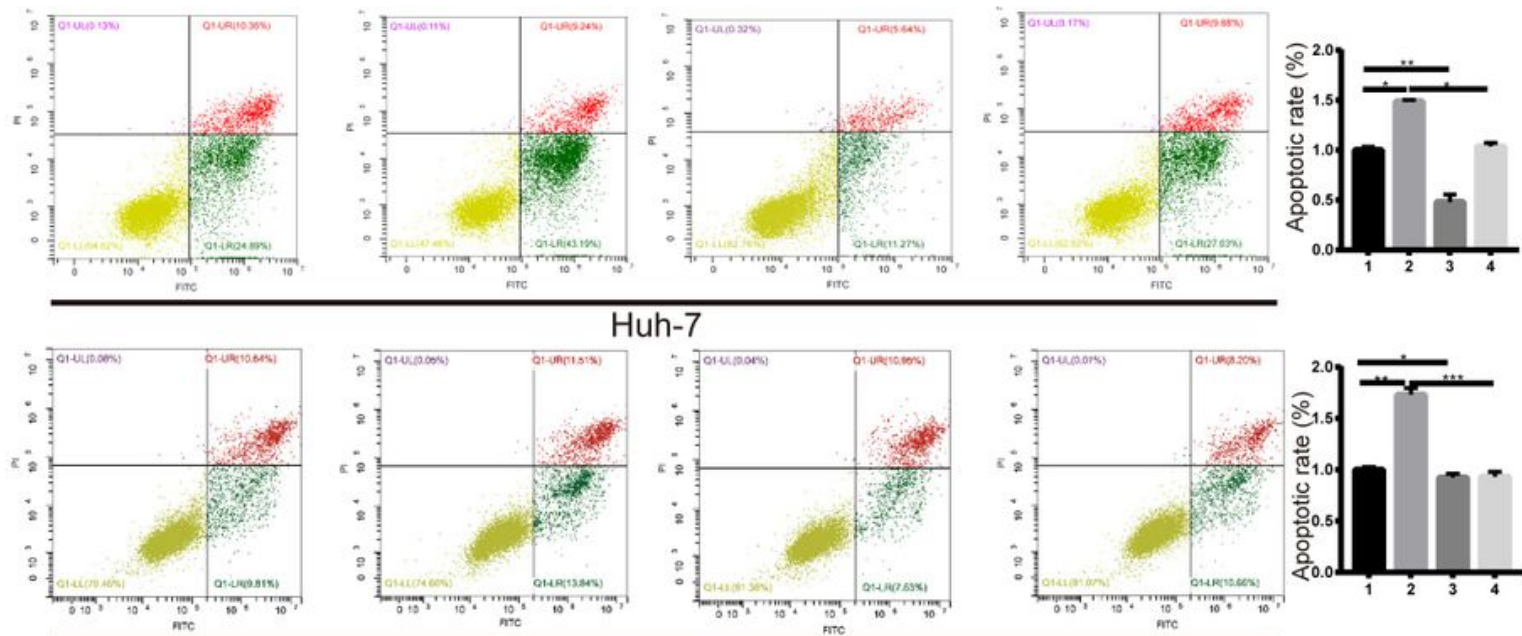

\section{Huh-7}

\section{B}
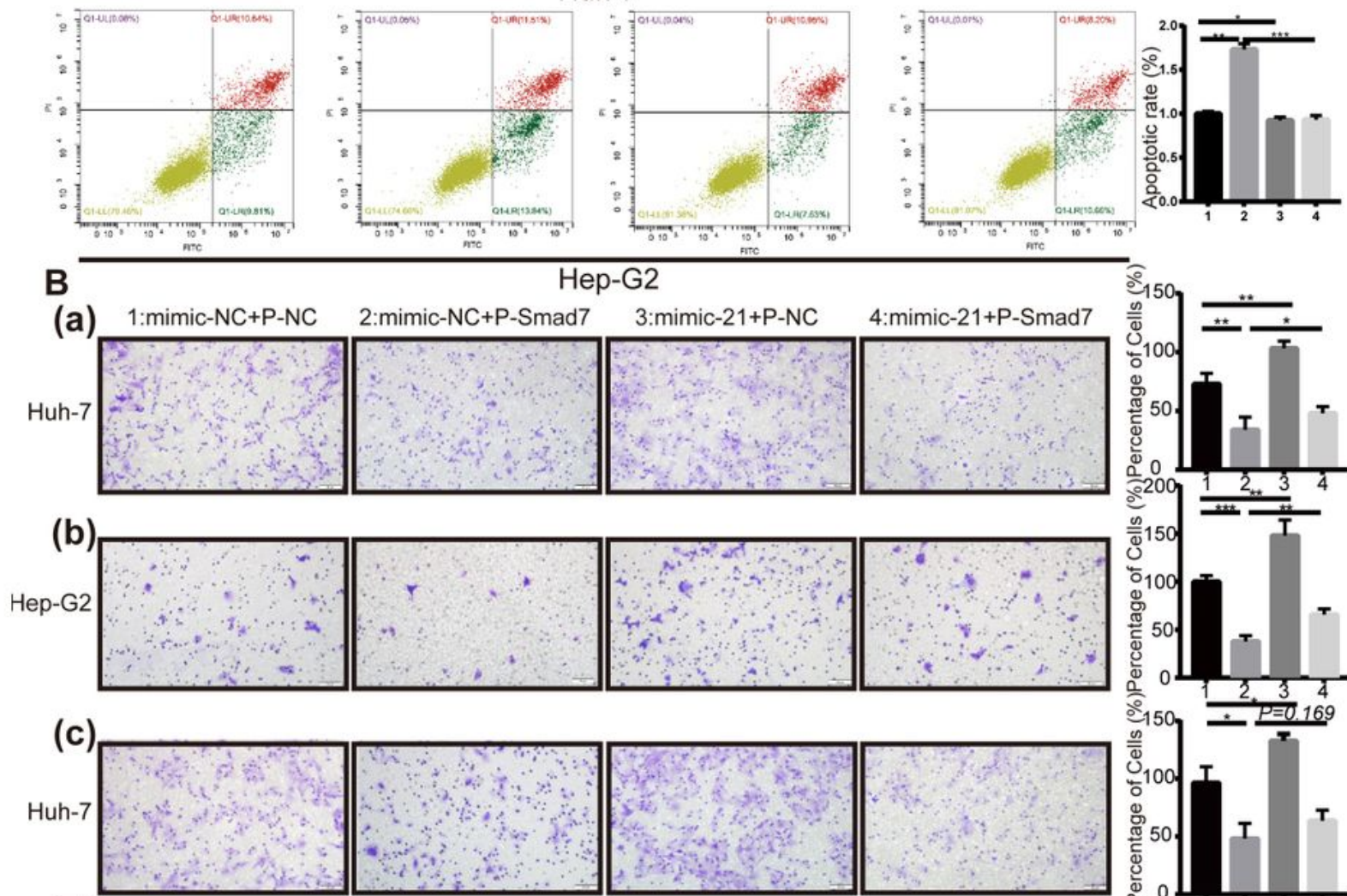

(d)
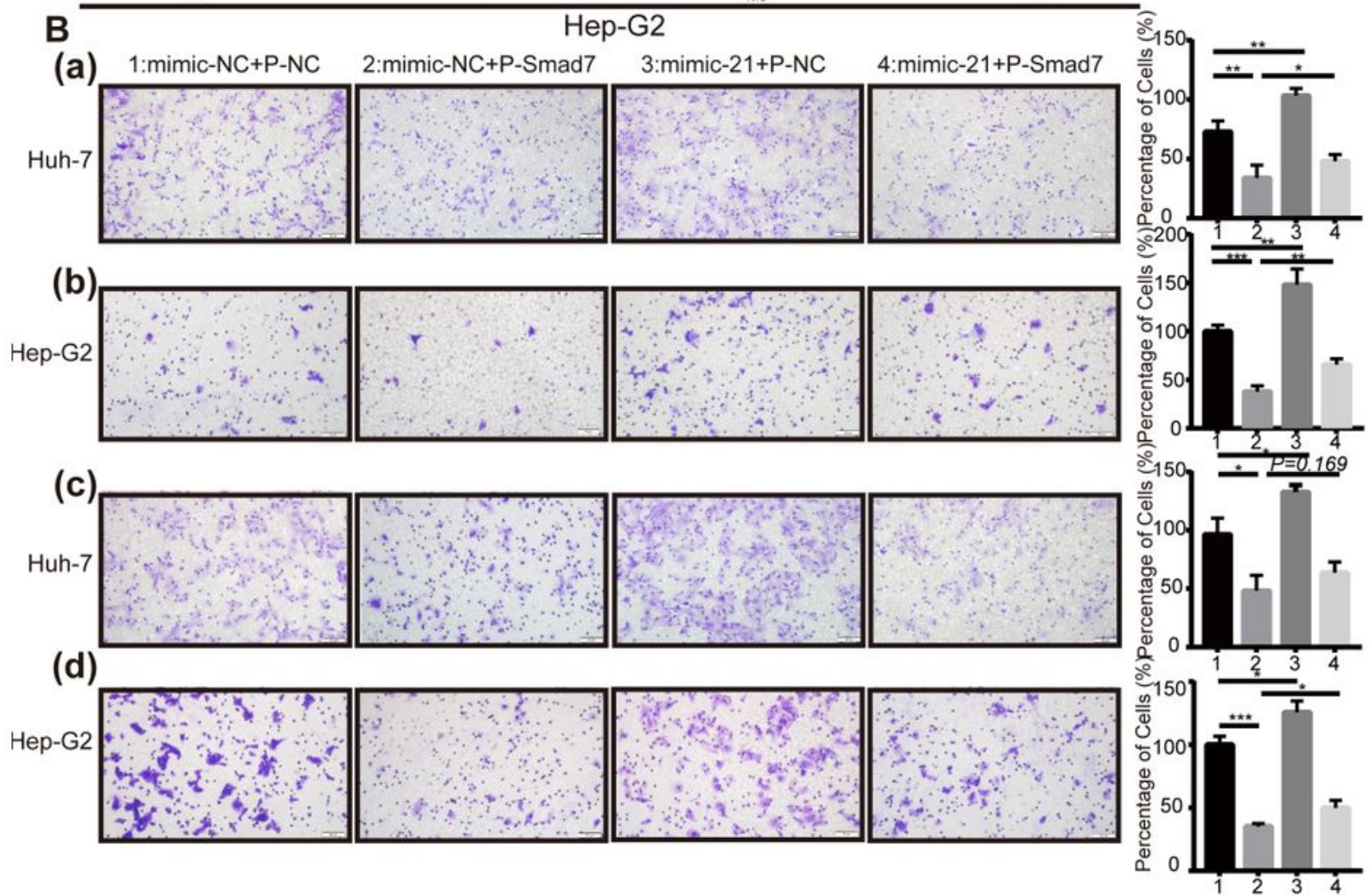

\section{Figure 4}

The effect of miR-21-3p on liver cancer cell malignant phenotypes could be partly reversed via overexpression of Smad7. (A) Flow cytometry was performed to analyze cell apoptosis index of Huh-7 and Hep-G2 cells after transfection. Cells were co-stained with Annexin FITC/PI, Annexin FITC+/PI- cells 
considered as early apoptotic cells were stained green. Normal, late apoptosis and necrosis cells were shown in yellow, red and pink, respectively. Data were shown as mean \pm SD from three independent experiments. (B) Transwell assay detected the migration (a, b) and invasion (c, d) ability of Huh-7 and Hep-G2 cells after transfection separately. * $P<0.05$, ** $P<0.01$; *** $P<0.001$.

A

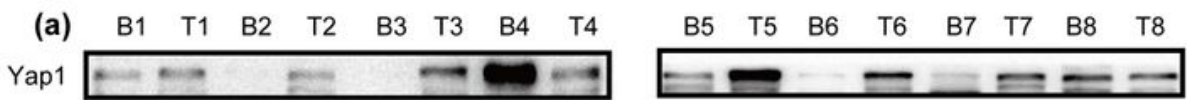

GAPDH

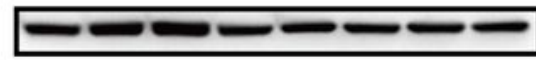

B

Yap1 $\longrightarrow-\longrightarrow$

${ }_{B}^{\mathrm{GAPDH}}=-\infty$

(a)
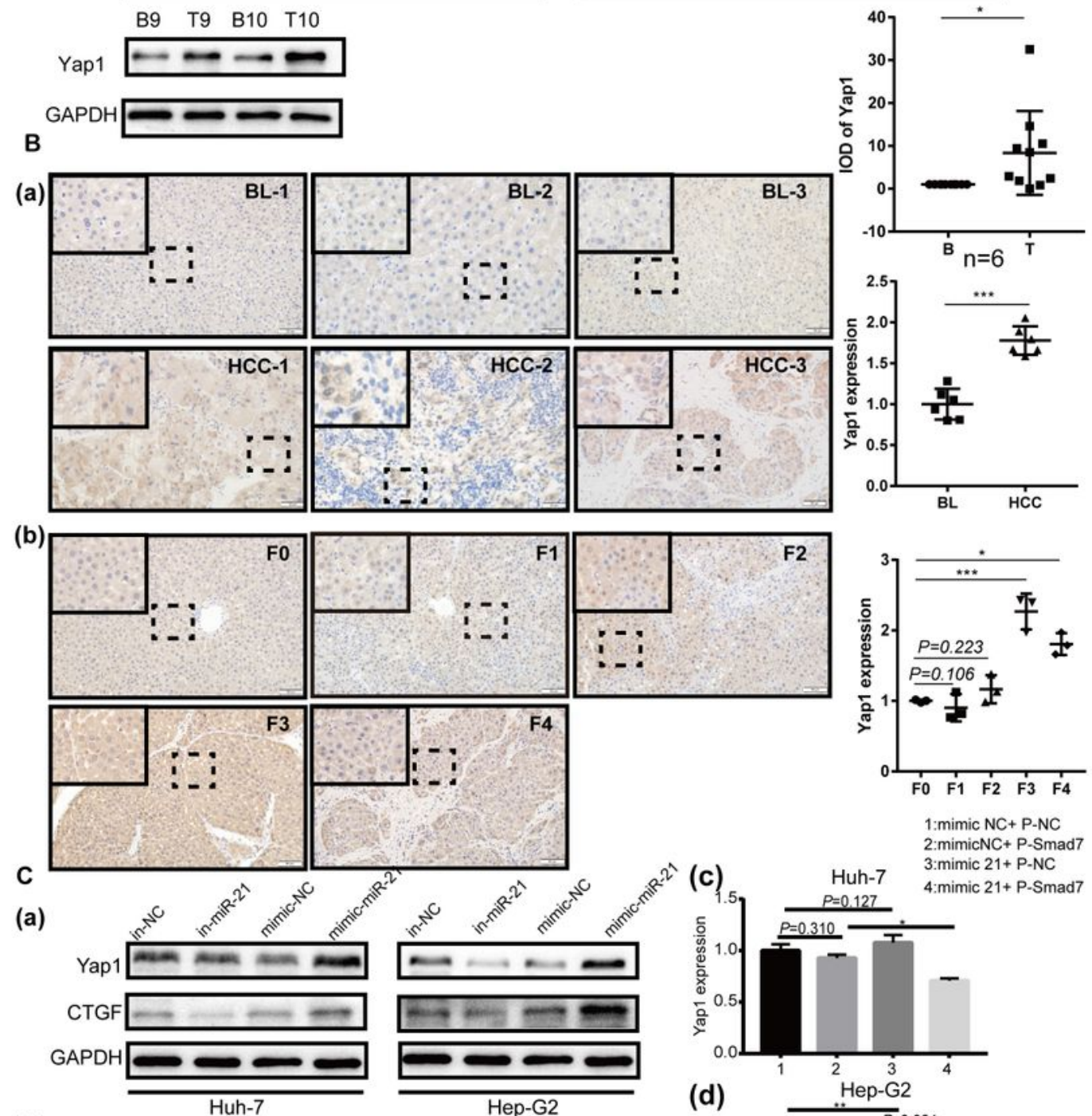

(b)

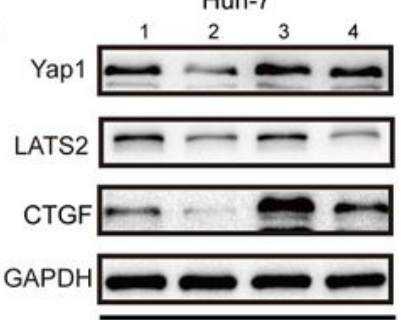

Huh-7
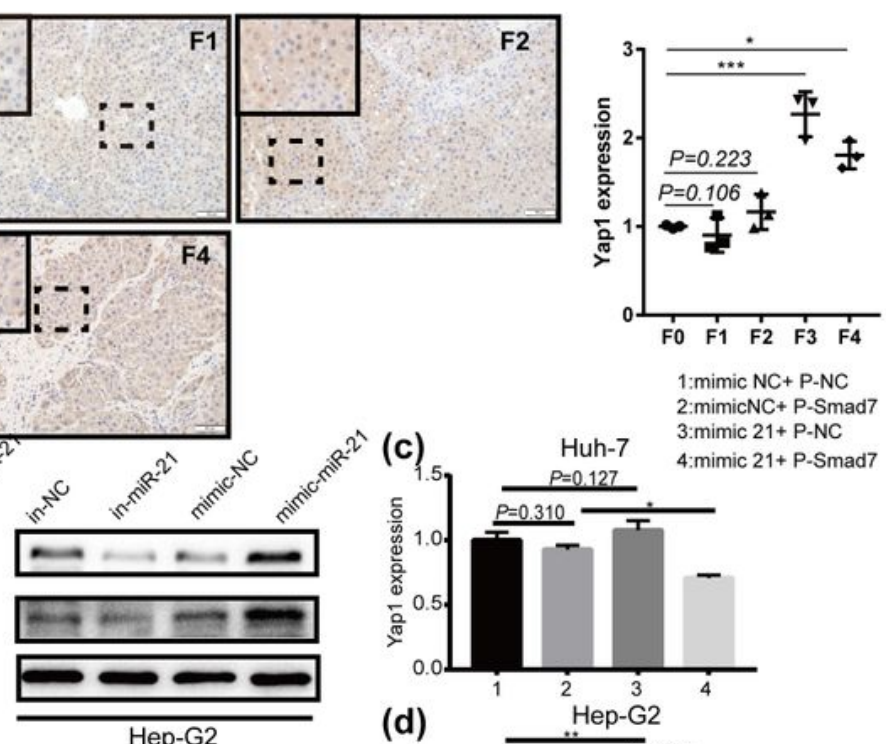

(d) Hep-G2

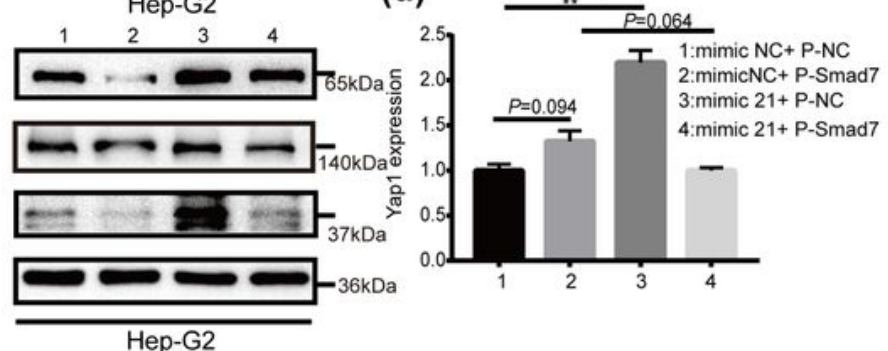

Figure 5 
Yap1 was up-regulated in HCCs, and could be facilitated by miR-21-3p via inhibiting Smad7 (A) Western blotting was performed to show Yap1's expressions in human HCCs (T: tumor) and their BLs (B:

background). (B) Expression of Yap1 expression in HCCs, BLs (a) and different liver disease stages in rat models (b) was checked through IHC staining assay (magnification 400x). Six paired human samples and three rats each group were calculated during statistical analysis. (C) Western blotting of Yap1 after 48h transfection with miR-21-3p inhibitors or mimics in Huh-7 cells and Hep-G2 cells (a), the protein expression level (b) and mRNA expression level (c,d) of Yap1,CTGF, LATS2 after transfecting plasmids Smad7 with/or miR-21-3p mimics. 
A

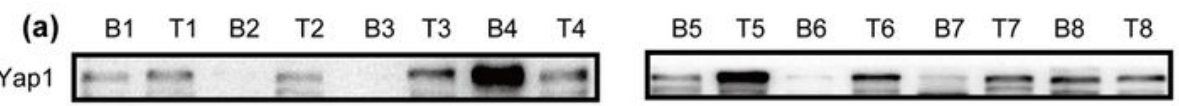
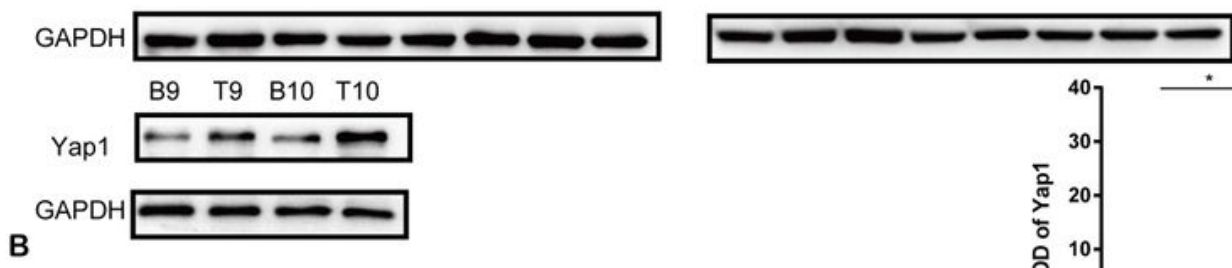

(a)
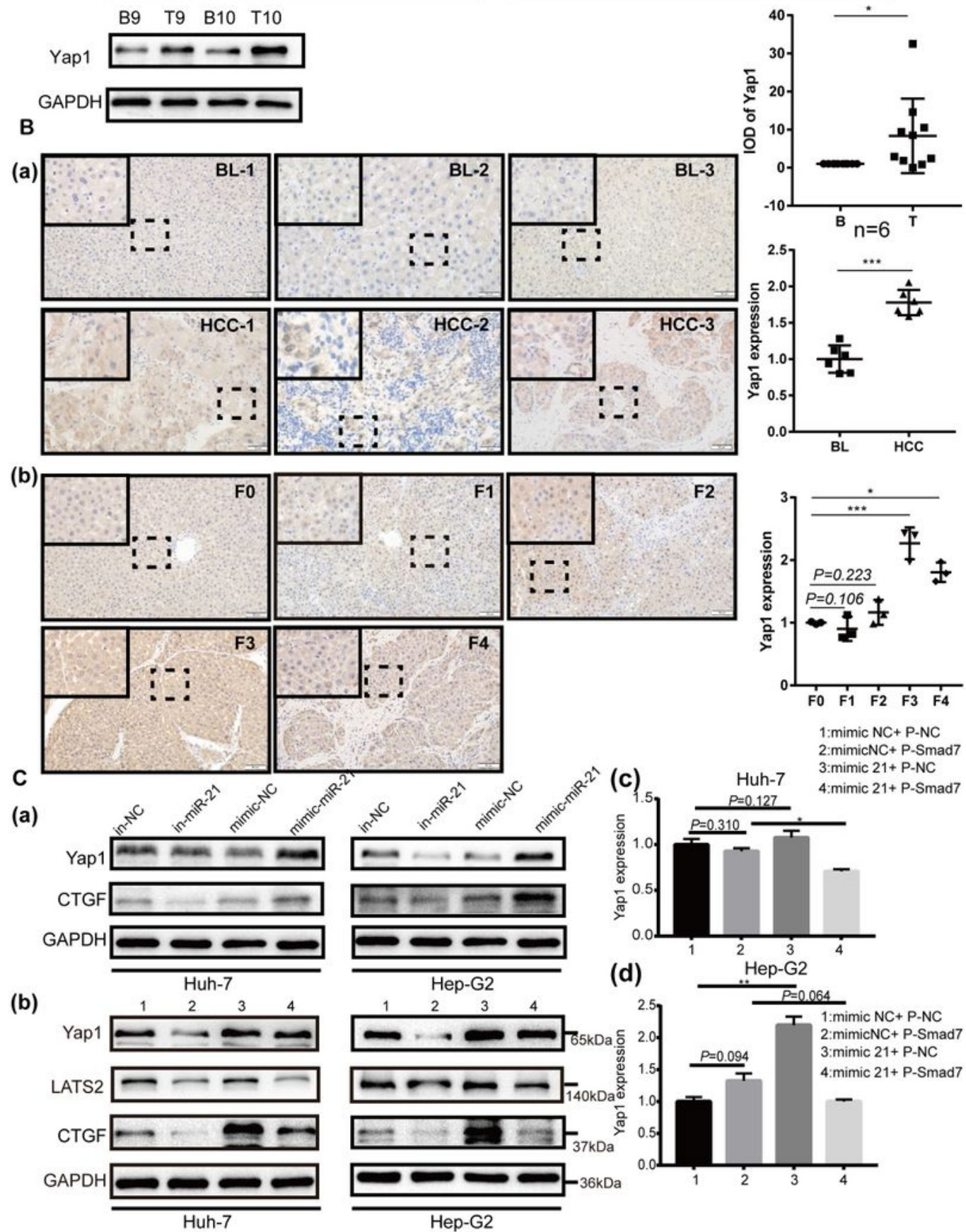

(a)
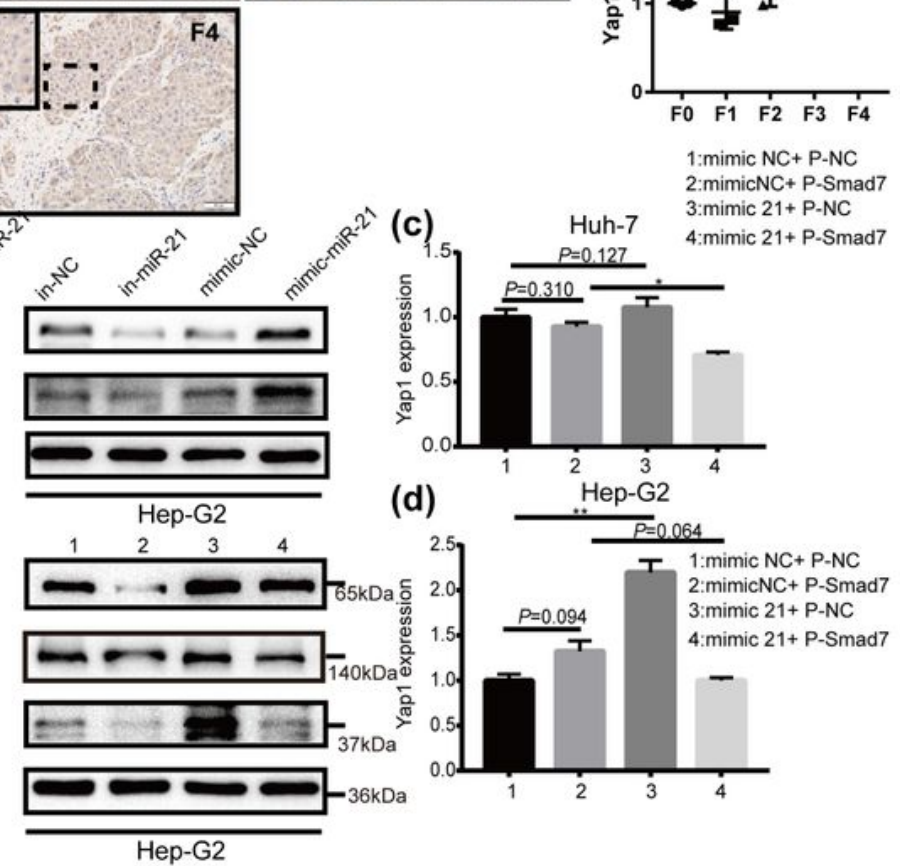

\section{Figure 5}

Yap1 was up-regulated in HCCs, and could be facilitated by miR-21-3p via inhibiting Smad7 (A) Western blotting was performed to show Yap1's expressions in human HCCs (T: tumor) and their BLs (B: background). (B) Expression of Yap1 expression in HCCs, BLs (a) and different liver disease stages in rat models (b) was checked through IHC staining assay (magnification 400x). Six paired human samples and three rats each group were calculated during statistical analysis. (C) Western blotting of Yap1 after 
48h transfection with miR-21-3p inhibitors or mimics in Huh-7 cells and Hep-G2 cells (a), the protein expression level (b) and mRNA expression level (c,d) of Yap1,CTGF, LATS2 after transfecting plasmids Smad7 with/or miR-21-3p mimics.
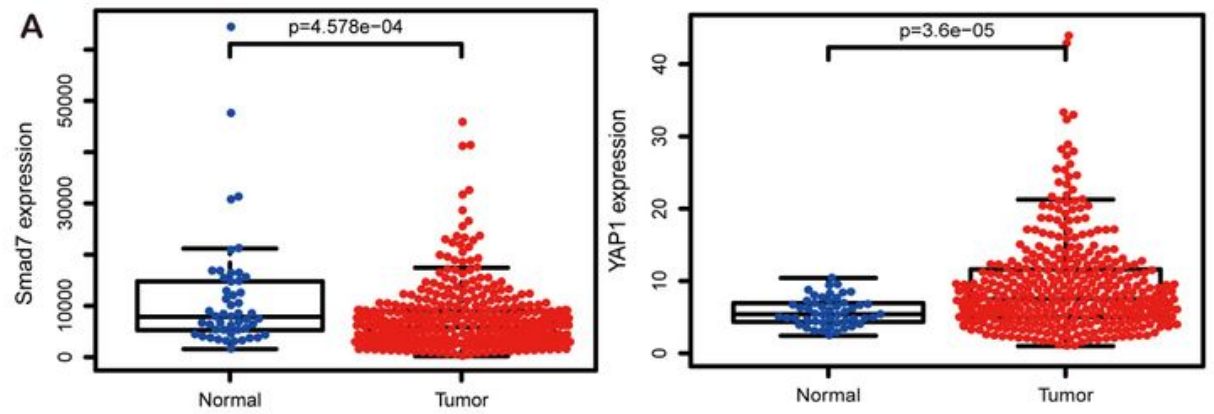

B
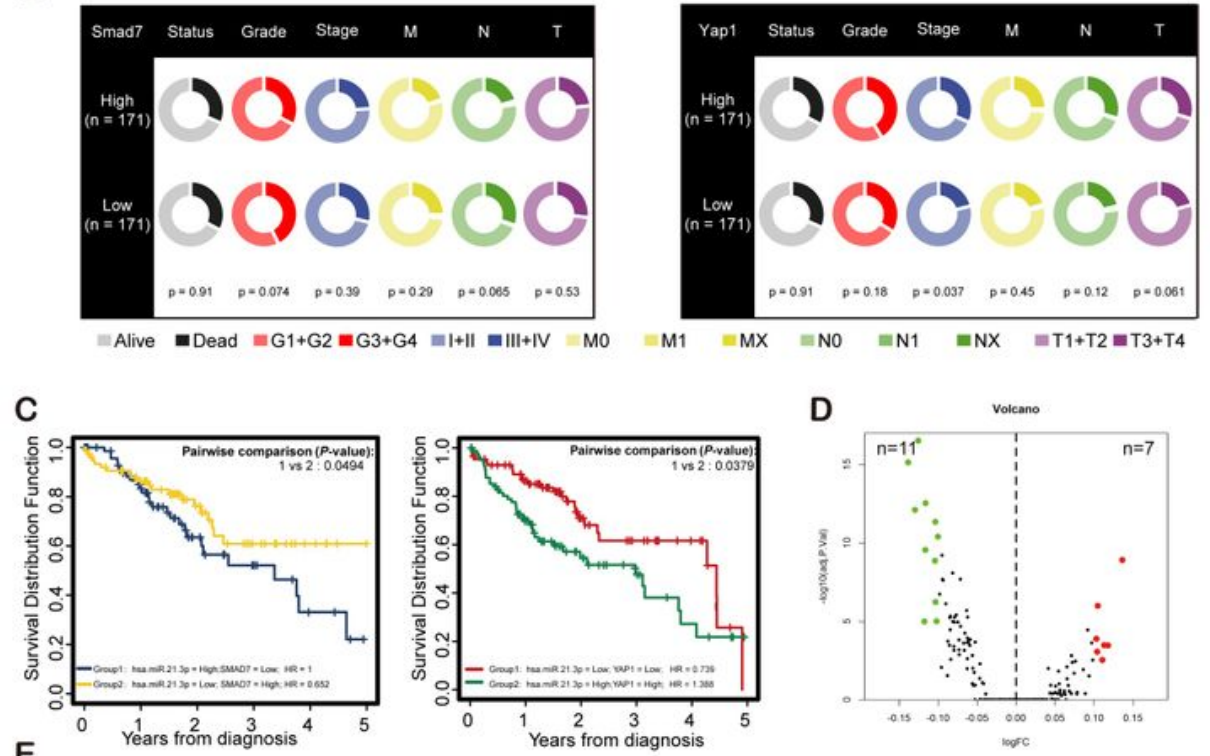

E

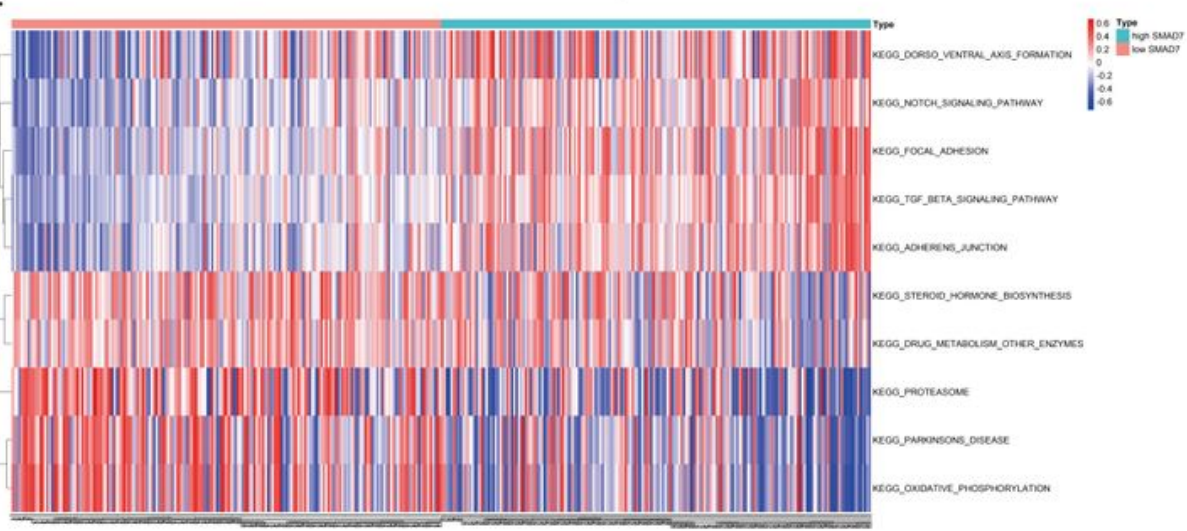

Figure 6

The clinical significance of Smad7/Yap1 in HCCs based on bioinformatics analysis. (A) Discrepancies of Smad7 (a) and Yap1 (b) expression levels in liver tumor tissues (Tumor) and non-paired relative normal 
samples (Normal) in the TCGA database were presented. (B) Relationship between the expression of Smad7, Yap1 and liver cancer clinicopathological terms using Wilcoxon rank-sum test. Data with incomplete clinical traits were excluded during analysis. (C) Kaplan-Meier curves representing the percent overall survival (OS) in HCC patients based on miR-21-3p /Smad7 or miR-21-3p/Yap1 expression levels in TCGA data set $(n=376)$. Statistical significance between miRNA/mRNA expression and OS was determined by the Log-rank test. Smad7-related KEGG pathways based on the gene set variation analysis of The Cancer Genome Atlas data. Shown in the volcano map (D) and heat map (E) (top ten including representative terms), respectively. 

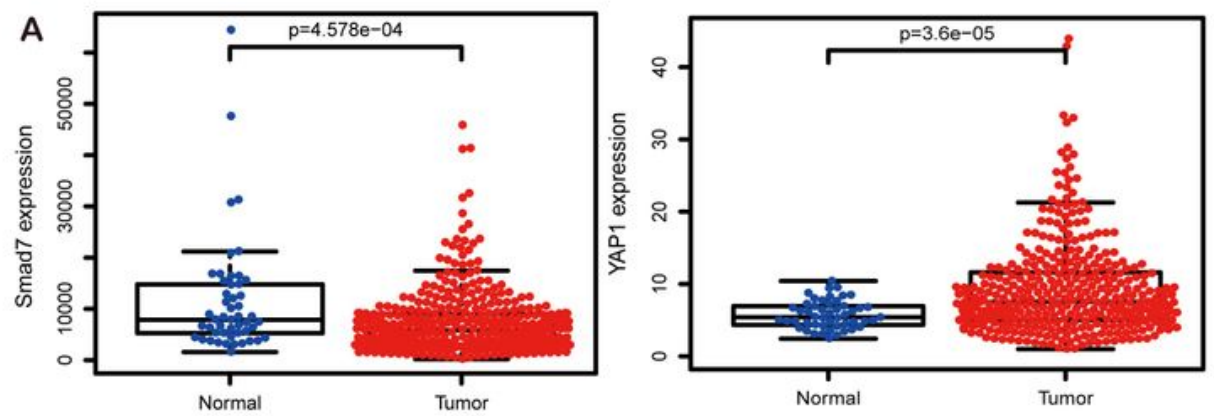

B
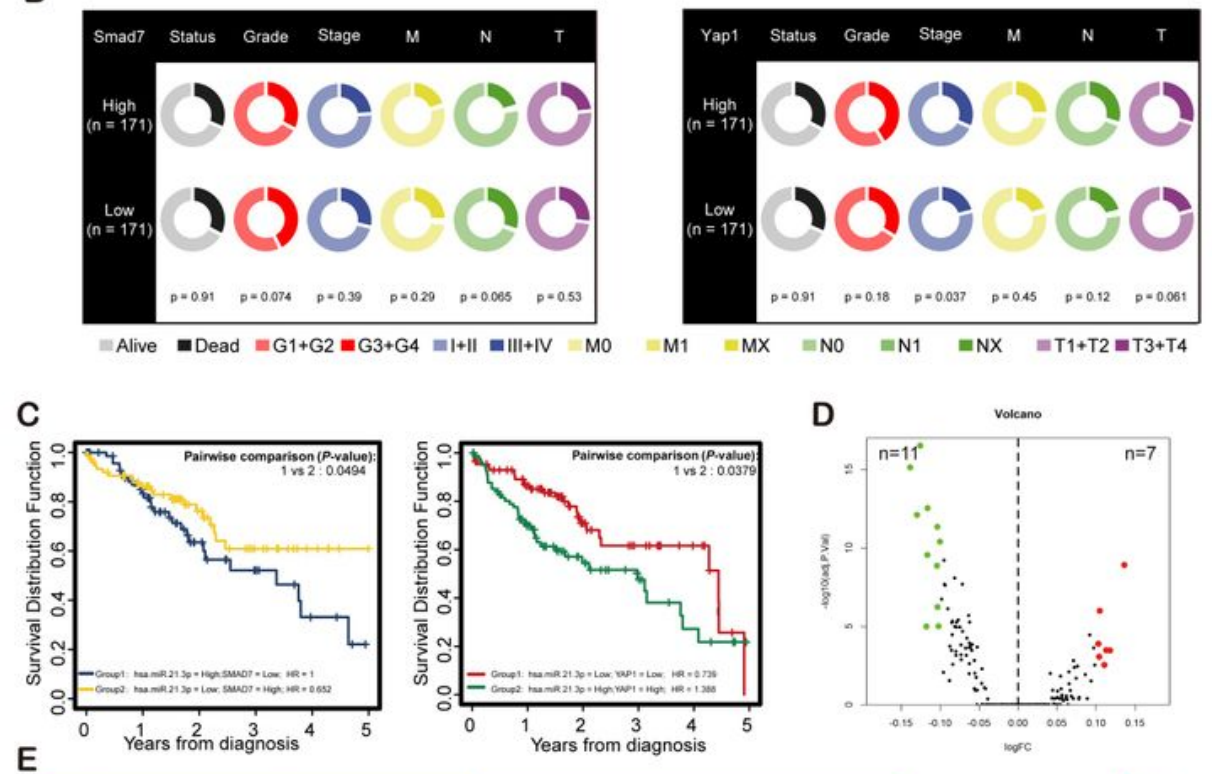

E

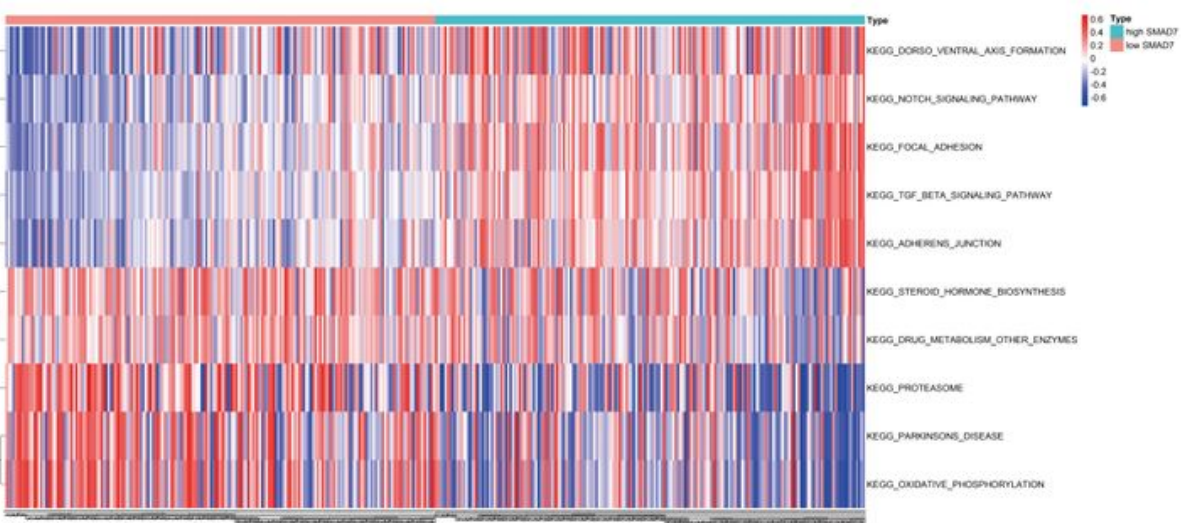

\section{Figure 6}

The clinical significance of Smad7/Yap1 in HCCs based on bioinformatics analysis. (A) Discrepancies of Smad7 (a) and Yap1 (b) expression levels in liver tumor tissues (Tumor) and non-paired relative normal samples (Normal) in the TCGA database were presented. (B) Relationship between the expression of Smad7, Yap1 and liver cancer clinicopathological terms using Wilcoxon rank-sum test. Data with incomplete clinical traits were excluded during analysis. (C) Kaplan-Meier curves representing the percent 
overall survival (OS) in HCC patients based on miR-21-3p /Smad7 or miR-21-3p/Yap1 expression levels in TCGA data set $(n=376)$. Statistical significance between miRNA/mRNA expression and OS was determined by the Log-rank test. Smad7-related KEGG pathways based on the gene set variation analysis of The Cancer Genome Atlas data. Shown in the volcano map (D) and heat map (E) (top ten including representative terms), respectively.

\section{Supplementary Files}

This is a list of supplementary files associated with this preprint. Click to download.

- FigureS1.tif

- FigureS1.tif

- FigureS2.tif

- Figures2.tif

- Figures3.tif

- FigureS3.tif

- Fig7.jpg

- Fig7.jpg 